\title{
Ulam-type stability for a class of implicit fractional differential equations with non-instantaneous integral impulses and boundary condition
}

Akbar Zada', Sartaj Ali ${ }^{1}$ and Yongjin $\mathrm{Li}^{2 *}$ (I)

"Correspondence:

stslyj@mail.sysu.edu.cn

${ }^{2}$ Department of Mathematics, Sun

Yat-sen University, Guangzhou,

510275, P.R. China

Full list of author information is

available at the end of the article

\section{Springer}

\begin{abstract}
In this paper, we investigate four different types of Ulam stability, i.e., Ulam-Hyers stability, generalized Ulam-Hyers stability, Ulam-Hyers-Rassias stability and generalized Ulam-Hyers-Rassias stability for a class of nonlinear implicit fractional differential equations with non-instantaneous integral impulses and nonlinear integral boundary condition. We also establish certain conditions for the existence and uniqueness of solutions for such a class of fractional differential equations using Caputo fractional derivative. The arguments are based on generalized Diaz-Margolis's fixed point theorem. We provide two examples, which shows the validity of our main results.
\end{abstract}

MSC: 26A33; 34A08; 34B27

Keywords: Caputo fractional derivative; implicit fractional differential equations; fractional integral; non-instantaneous impulses; Ulam-type stability; Diaz-Margolis's fixed point theorem

\section{Introduction}

Fractional calculus was originated from a question of L'Hospital, in which he asked about the generalization of integral order differentiation. At the end of 17th century, Leibnitz used a notation $\frac{d^{m}}{d x^{m}} g(t)$ for $m t h$ integral order derivative of a function $g(t)$. At that time L'Hospital asked a question, "What should happen if the order is $\frac{1}{2}$ ?". Leibnitz on 30th September 1695 (the exact beginning of the fractional calculus) replied, "It will be a paradox, from which later useful consequences will be drawn" [1]. After that, in 1819, Lacroix introduced fractional order derivative [2].

Fractional calculus is as old as the conventional calculus, and it is the generalization of integral order differentiation and integration to arbitrary non-integer order. For detailed study, see the books such as [3-7] and the papers $[8,9]$. The attraction towards this subject is due to the fact that fractional derivatives and integrals are not a local property. That is why fractional differential and integral models captured the reality of the nature better, as these models considered the history and nonlocal distributed effects; for details, see the monographs [10-12]. Fractional calculus has a large number of applications in different

(c) The Author(s) 2017. This article is distributed under the terms of the Creative Commons Attribution 4.0 International License (http://creativecommons.org/licenses/by/4.0/), which permits unrestricted use, distribution, and reproduction in any medium, provided you give appropriate credit to the original author(s) and the source, provide a link to the Creative Commons license, and indicate if changes were made. 
branches of science, engineering as well as in medical fields. The fractional differential models describe many real world phenomena in different fields, i.e., biology, dynamical systems, physics, control theory, chemistry and in many other fields, in a more efficient and realistic way.

In recent research work, several researchers of mathematics community studied impulsive fractional differential equations due to their applications in different fields of engineering and medical sciences [7, 10-12]. Impulsive fractional differential equations are used to describe real world phenomena like evolutionary processes characterized by abrupt changes of the state at certain instants and many others. The monographs [13-16] treated fractional differential equations with instantaneous impulses of the following form:

$$
\begin{cases}{ }^{c} D_{0, t}^{\alpha} v(t)=u(t, v(t)), & t \in[0, T] \backslash\left\{t_{1}, t_{2}, \ldots, t_{m}\right\}, \\ \Delta v(t)=I_{k}\left(v\left(t_{k}^{-}\right)\right), & k=1,2, \ldots, m,\end{cases}
$$

where ${ }^{c} D_{0, t}^{\alpha}$ is the Caputo fractional derivative of order $\alpha \in(n-1, n), n$ is any natural number with lower bound $0, u:[0, T] \times \mathbb{R} \rightarrow \mathbb{R}$ is continuous, $I_{k}: \mathbb{R} \rightarrow \mathbb{R}$ are instantaneous impulses and $t_{k}$ satisfies $0=t_{0}<t_{1}<\cdots<t_{m}=T, T>0, v\left(t_{k}^{+}\right)=\lim _{\epsilon \rightarrow 0^{+}} v\left(t_{k}+\epsilon\right)$ and $v\left(t_{k}^{-}\right)=\lim _{\epsilon \rightarrow 0^{-}} v\left(t_{k}+\epsilon\right)$ denotes the right and left limits of $v(t)$ at $t=t_{k}$, respectively.

In [17] Mardanov et al. considered impulsive fractional differential equations with two points integral boundary conditions of the following form:

$$
\begin{cases}{ }^{c} D_{0^{+}}^{\xi} p(t)=q(t, p(t)), & t \in[0, T] \backslash\left\{t_{1}, t_{2}, \ldots, t_{r}\right\}, \\ p\left(t_{k}^{+}\right)-p\left(t_{k}^{-}\right)=I_{k}\left(p\left(t_{k}\right)\right), & k=1,2, \ldots, r, \\ A p(0)+B p(T)=\int_{0}^{T} f(s, p(s)) d s, & \end{cases}
$$

where $A, B \in \mathbb{R}^{m \times m}$ are given matrices and $\operatorname{det}(A+B) \neq 0$. Here $q, f:[0, T] \times \mathbb{R}^{m} \rightarrow \mathbb{R}^{m}$ and $I_{i}: \mathbb{R}^{m} \rightarrow \mathbb{R}^{m}$ are given functions.

Problems (1.1) and (1.2) do not characterize completely the process like hemodynamic equilibrium of a person, i.e., the introduction of the drugs in the bloodstream and the consequent absorption for the body are gradual and continuous processes. Such situations are characterized by non-instantaneous impulses, which begin from an arbitrary fixed point and stay active on a finite time interval.

Motivated by the above-mentioned work, we consider nonlinear implicit fractional differential equations with non-instantaneous integral impulses and nonlinear integral boundary condition in the following form:

$$
\begin{cases}{ }^{c} D_{0, t}^{\beta} y(t)=f\left(t, y(t),{ }^{c} D_{0, t}^{\beta} y(t)\right), & t \in\left(t_{k}, s_{k}\right], k=0,1,2, \ldots, m, \beta \in(0,1] \\ y(t)=I_{s_{k-1}, t_{k}}^{\beta}\left(\xi_{k}(t, y(t))\right), & t \in\left(s_{k-1}, t_{k}\right], k=1,2, \ldots, m, \\ y(0)=I_{0, T}^{\beta} \eta(t, y(t)), & \end{cases}
$$

where ${ }^{c} D_{0, t}^{\beta}$ is the Caputo fractional derivative of order $\beta$ with lower limit $0,0=t_{0}<s_{0}<$ $t_{1}<s_{1}<\cdots<t_{m}<s_{m}=T, T>0$ is pre-fixed number. The functions $f:[0, T] \times \mathbb{R} \times \mathbb{R} \rightarrow \mathbb{R}$, $\eta:[0, T] \times \mathbb{R} \rightarrow \mathbb{R}$ are continuous and $\xi_{k}:\left[s_{k-1}, t_{k}\right] \times \mathbb{R} \rightarrow \mathbb{R}$ is also continuous for all $k=1,2, \ldots, m$, which is known as non-instantaneous impulses. The notations $I_{s_{k-1}, t_{k}}^{\beta}$ and $I_{0, T}^{\beta}$ are given to fractional integrals of order $\beta$ with limits $s_{k-1}$ to $t_{k}$ and 0 to $T$, respectively, 
and

$$
I_{0, T}^{\beta} \eta(t, y(t))=\frac{1}{\Gamma(\beta)} \int_{0}^{T}(T-s)^{\beta-1} \eta(s, y(s)) d s .
$$

Since 1940, Ulam-type stability problems [18] have been studied by a large number of mathematicians. This stability analysis is very useful in many applications, such as numerical analysis, optimization, etc., where finding the exact solution is quite difficult. For detailed study of Ulam-type stability with different approaches, we recommend papers such as [19-33].

The aim of this paper is to establish sufficient conditions for the existence, uniqueness and Ulam-type stability for a class of nonlinear implicit fractional differential equations with non-instantaneous integral impulses and nonlinear integral boundary condition, via generalized Diaz-Margolis's fixed point theorem. This paper is presented as follows. Section 2 contains some basic definitions, notations and lemmas regarding fractional differential equations. Section 3 contains the framework of the solutions for problem (1.3). In Section 4 we show some existence and uniqueness conditions, along with different types of Ulam stability for problem (1.3). Section 5 contains some examples.

\section{Preliminaries}

In this section we gather some basic facts, definitions and lemmas regarding fractional differential equations, which we utilized throughout this paper, to obtain our main results.

Definition 2.1 ([34]) An arbitrary order fractional integral of a function $\delta \in L^{1}\left([0, T], \mathbb{R}_{+}\right)$ of order $\beta \in \mathbb{R}_{+}$is defined as

$$
I_{0, t}^{\beta} \delta(t)=\frac{1}{\Gamma(\beta)} \int_{0}^{t}(t-s)^{\beta-1} \delta(s) d s, \quad t>0,
$$

where $\Gamma$ is the Euler gamma function defined by $\Gamma(\beta)=\int_{0}^{\infty} p^{\beta-1} e^{-p} d p$.

Definition 2.2 ([34]) The Caputo fractional derivative of order $\beta \in \mathbb{R}_{+}$, for a function $\delta:[0, T] \rightarrow \mathbb{R}$, is defined as

$$
\left({ }^{c} D_{0, t}^{\beta} \delta\right)(t)=\frac{1}{\Gamma(n-\beta)} \int_{0}^{t}(t-s)^{n-\beta-1} \delta^{(n)}(s) d s, \quad n=[\beta]+1,
$$

where $[\beta]$ denotes the integer part of the real number $\beta$.

Lemma 2.3 ([34]) For a nonnegative value of $\beta$, we have

$$
I_{0, t}^{\beta}\left({ }^{c} D_{0, t}^{\beta} \delta(t)\right)=\delta(t)-\sum_{m=0}^{n-1} \frac{\delta^{m}(0)}{m !} t^{m}, \quad n=[\beta]+1 .
$$

Lemma 2.4 ([34]) For $\beta>0$, the Caputo fractional differential equation ${ }^{c} D_{0, t}^{\beta} \delta(t)=0$ has a solution of the following form:

$$
\delta(t)=a_{0}+a_{1} t+a_{2} t^{2}+\cdots+a_{n-1} t^{n-1},
$$

where $a_{i} \in \mathbb{R}, i=0,1, \ldots, n-1$ and $n=[\beta]+1$. 
Lemma 2.5 ([34]) For $\beta>0$, we have

$$
I_{0, t}^{\beta}\left({ }^{c} D_{0, t}^{\beta} \delta(t)\right)=\delta(t)+a_{0}+a_{1} t+a_{2} t^{2}+\cdots+a_{n-1} t^{n-1},
$$

where $a_{i} \in \mathbb{R}, i=0,1, \ldots, n-1$ and $n=[\beta]+1$.

Let $J=[0, T]$ and $C(J, \mathbb{R})$ be the space of all continuous functions from $J$ to $\mathbb{R}$. Let $\hat{\mathfrak{B}}=$ $P C(J, \mathbb{R})$ represent the space of piecewise continuous functions. Obviously, $\hat{\mathfrak{B}}=P C(J, \mathbb{R})$ is a Banach space with the norm

$$
\|y\|_{\hat{\mathfrak{B}}}=\sup _{t \in J}\{|y|\} .
$$

Now, we introduce the concept of Ulam-type stabilities for problem (1.3).

Let $x \in \hat{\mathfrak{B}}, \varepsilon>0, \psi>0, \lambda \geq 0$ and a nondecreasing function $\phi \in C\left(J, \mathbb{R}_{+}\right)$. Let us consider the following set of inequalities:

$$
\begin{aligned}
& \begin{cases}\left|{ }^{c} D_{0, t}^{\beta} x(t)-f\left(t, x(t),{ }^{c} D_{0, t}^{\beta} x(t)\right)\right| \leq \varepsilon, & t \in\left(t_{k}, s_{k}\right], k=0,1,2, \ldots, m, \beta \in(0,1], \\
\left|x(t)-I_{s_{k-1}, t_{k}}^{\beta}\left(\xi_{k}(t, y(t))\right)\right| \leq \varepsilon, & t \in\left(s_{k-1}, t_{k}\right], k=1,2, \ldots, m,\end{cases} \\
& \begin{cases}\left|{ }^{c} D_{0, t}^{\beta} x(t)-f\left(t, x(t),{ }^{c} D_{0, t}^{\beta} x(t)\right)\right| \leq \phi(t), & t \in\left(t_{k}, s_{k}\right], k=0,1,2, \ldots, m, \beta \in(0,1], \\
\left|x(t)-I_{s_{k-1}, t_{k}}^{\beta}\left(\xi_{k}(t, y(t))\right)\right| \leq \psi, & t \in\left(s_{k-1}, t_{k}\right], k=1,2, \ldots, m,\end{cases}
\end{aligned}
$$

and

$$
\begin{cases}\left|{ }^{c} D_{0, t}^{\beta} x(t)-f\left(t, x(t),{ }^{c} D_{0, t}^{\beta} x(t)\right)\right| \leq \varepsilon \phi(t), & t \in\left(t_{k}, s_{k}\right], k=0,1,2, \ldots, m, \beta \in(0,1] \\ \left|x(t)-I_{s_{k-1}, t_{k}}^{\beta}\left(\xi_{k}(t, y(t))\right)\right| \leq \varepsilon \psi, & t \in\left(s_{k-1}, t_{k}\right], k=1,2, \ldots, m .\end{cases}
$$

Definition 2.6 Problem (1.3) is Ulam-Hyers stable if there exists a real constant $c_{f, \beta, g}>0$ such that, for given $\varepsilon>0$ and for each solution $x \in \hat{\mathfrak{B}}$ of inequality (2.1), there exists a solution $y \in \hat{\mathfrak{B}}$ of problem (1.3) with

$$
|x(t)-y(t)| \leq c_{f, \beta, g} \varepsilon, \quad t \in J .
$$

Definition 2.7 Problem (1.3) is generalized Ulam-Hyers stable if there exists a function $\lambda_{f, \beta, g} \in C\left(\mathbb{R}_{+}, \mathbb{R}_{+}\right), \lambda_{f, \beta, g}(0)=0$ such that, for given $\varepsilon>0$ and for each solution $x \in \hat{\mathfrak{B}}$ of inequality (2.1), there exists a solution $y \in \hat{\mathfrak{B}}$ of problem (1.3) with

$$
|x(t)-y(t)| \leq \lambda_{f, \beta, g}(\varepsilon), \quad t \in J
$$

Definition 2.8 Problem (1.3) is Ulam-Hyers-Rassias stable with respect to $(\phi, \psi)$ if there exists a real constant $c_{f, \beta, g, \psi}>0$ such that, for given $\varepsilon>0$ and for each solution $x \in \hat{\mathfrak{B}}$ of inequality (2.3), there exists a solution $y \in \hat{\mathfrak{B}}$ of problem (1.3) with

$$
|x(t)-y(t)| \leq c_{f, \beta, g, \psi} \varepsilon(\phi(t)+\psi), \quad t \in J .
$$


Definition 2.9 Problem (1.3) is generalized Ulam-Hyers-Rassias stable with respect to $(\phi, \psi)$ if there exists a real constant $c_{f, \beta, g, \psi}>0$ such that, for each solution $x \in \hat{\mathfrak{B}}$ of inequality (2.2), there exists a solution $y \in \hat{\mathfrak{B}}$ of problem (1.3) with

$$
|x(t)-y(t)| \leq c_{f, \beta, g, \psi}(\phi(t)+\psi), \quad t \in J .
$$

Remark 2.10 From the above definitions clearly we see that: (1) Definition 2.6 implies Definition 2.7 if $\lambda_{f, \beta, g}(\varepsilon)=c_{f, \beta, g} \varepsilon, \lambda_{f, \beta, g}(0)=0$; (2) Definition 2.8 for $\varepsilon=1$ implies Definition 2.9; (3) Definition 2.8 for $\phi(t)=\psi=1$ implies Definition 2.6.

Remark 2.11 A function $x \in \hat{\mathfrak{B}}$ is a solution of inequality (2.1) if there exist a function $Q \in \hat{\mathfrak{B}}$ and an $x$-dependent sequence $Q_{k}, k=1,2, \ldots, m$, such that

- $|Q(t)| \leq \varepsilon, t \in J$.

- $\left|Q_{k}\right| \leq \varepsilon, k=1,2, \ldots, m$.

- ${ }^{C} D_{0, t}^{\beta} x(t)=f\left(t, x(t),{ }^{C} D_{0, t}^{\beta} x(t)\right)+Q(t), t \in\left(t_{k}, s_{k}\right], k=0,1, \ldots, m$.

- $x(t)=I_{s_{k-1}, t_{k}}^{\beta}\left(\xi_{k}(t, x(t))\right)+Q_{k}, t \in\left(s_{k-1}, t_{k}\right], k=1,2, \ldots, m$.

Remark 2.12 A function $x \in \hat{\mathfrak{B}}$ is a solution of inequality (2.2) if there exist a function $Q \in \hat{\mathfrak{B}}$ and a sequence $Q_{k}, k=1,2, \ldots, m$, which depends on $x$, such that

- $|Q(t)| \leq \phi(t), t \in J$.

- $\left|Q_{k}\right| \leq \psi, k=1,2, \ldots, m$.

- ${ }^{C} D_{0, t}^{\beta} x(t)=f\left(t, x(t),{ }^{C} D_{0, t}^{\beta} x(t)\right)+Q(t), t \in\left(t_{k}, s_{k}\right], k=0,1, \ldots, m$.

- $x(t)=I_{s_{k-1}, t_{k}}^{\beta}\left(\xi_{k}(t, x(t))\right)+Q_{k}, t \in\left(s_{k-1}, t_{k}\right], k=1,2, \ldots, m$.

Remark 2.13 A function $x \in \hat{\mathfrak{B}}$ is a solution of inequality (2.3) if there exist a function $Q \in \hat{\mathfrak{B}}$ and a sequence $Q_{k}, k=1,2, \ldots, m$, which depends on $x$, such that

- $|Q(t)| \leq \varepsilon \phi(t), t \in J$.

- $\left|Q_{k}\right| \leq \varepsilon \psi, k=1,2, \ldots, m$.

- ${ }^{C} D_{0, t}^{\beta} x(t)=f\left(t, x(t),{ }^{C} D_{0, t}^{\beta} x(t)\right)+Q(t), t \in\left(t_{k}, s_{k}\right], k=0,1, \ldots, m$.

- $x(t)=I_{s_{k-1}, t_{k}}^{\beta}\left(\xi_{k}(t, x(t))\right)+Q_{k}, t \in\left(s_{k-1}, t_{k}\right], k=1,2, \ldots, m$.

In order to use a fixed point approach for contractions on a generalized complete metric space to obtain our main results, we need the following result.

Definition 2.14 ([35] (Generalized metric space)) Let $Y$ be a nonempty set, a function $d: Y \times Y \rightarrow[0, \infty]$ is said to be a generalized complete metric on $Y$ if and only if for all $u, v, w \in Y$ the following conditions hold:

- $d(u, v) \geq 0$,

- $d(u, v)=0$ if and only if $u=v$,

- $d(u, v)=d(v, u)$,

- $d(u, v) \leq d(u, w)+d(w, v)$.

Definition 2.15 ([35] (Generalized complete metric space)) Let $Y$ be a generalized metric space. If every $d$-Cauchy sequence in $Y$ is $d$-convergent, then $Y$ is called generalized complete metric space, i.e., if $\left\{a_{n}\right\}$ is a sequence in $Y$ such that $\lim _{m, n \rightarrow \infty} d\left(a_{n}, a_{m}\right)=0$, then there exists $u \in Y$ such that $\lim _{n \rightarrow \infty} d\left(a_{n}, u\right)=0$. 
Theorem 2.16 ([36] (Generalized Diaz-Margolis's fixed point theorem)) Suppose that $(Y, d)$ is a generalized complete metric space, and an operator $\Lambda: Y \rightarrow Y$ is strictly contractive with Lipschitz constant $L<1$. If there is an integer $m \geq 0$ such that $d\left(\Lambda^{m+1} x, \Lambda^{m} x\right)<\infty$ for some $x \in Y$, then we have the following:

(a) The sequence $\left\{\Lambda^{n} x\right\}$ converges to a fixed point $x^{*}$ of $\Lambda$.

(b) $x^{*}$ is the unique fixed point of $\Lambda$ in $Y^{*}=\left\{y \in Y \mid d\left(\Lambda^{m} x, y\right)<\infty\right\}$.

(c) If $y \in Y^{*}$, then $d\left(y, x^{*}\right) \leq \frac{1}{1-L} d(\Lambda y, y)$.

\section{The solution of a linear impulsive fractional boundary value problem}

In this section we find the solution of a linear impulsive problem, which is the corresponding linear form of problem (1.3), using Lemma 2.5. Consider a linear form of problem (1.3), i.e.,

$$
\begin{cases}{ }^{c} D_{0, t}^{\beta} y(t)=f(t), & t \in\left(t_{k}, s_{k}\right], k=0,1,2, \ldots, m, \beta \in(0,1] \\ y(t)=I_{s_{k-1}, t_{k}}^{\beta}\left(\xi_{k}(t)\right), & t \in\left(s_{k-1}, t_{k}\right], k=1,2, \ldots, m, \\ y(0)=I_{0, T}^{\beta} \eta(t, y(t)), & \end{cases}
$$

where ${ }^{c} D_{0, t}^{\beta}$ is the Caputo fractional derivative (CDF) of order $\beta$ with lower limit $0,0=t_{0}<$ $s_{0}<t_{1}<s_{1}<\cdots<t_{m}<s_{m}=T, T>0$ is pre-fixed number, $f: J \rightarrow \mathbb{R}$ is a given continuous function, $\xi_{k}:\left[s_{k-1}, t_{k}\right] \rightarrow \mathbb{R}, k=1,2, \ldots, m$, are non-instantaneous impulses, $\eta:[0, T] \times$ $\mathbb{R} \rightarrow \mathbb{R}$ is also a given continuous function, $I_{s_{k-1}, t_{k}}^{\beta}$ and $I_{0, T}^{\beta}$ are fractional integrals of order $\beta$ with limits $s_{k-1}$ to $t_{k}$ and 0 to $T$, respectively.

Lemma 3.1 Let $\beta \in(0,1]$ and $f: J \rightarrow \mathbb{R}$ be a given continuous function. A function $y \in \hat{\mathfrak{B}}$ is a solution of problem (3.1) if and only if $y \in \hat{\mathfrak{B}}$ is a solution of the following integral equation:

$$
y(t)= \begin{cases}\frac{1}{\Gamma(\beta)} \int_{0}^{t}(t-s)^{\beta-1} f(s) d s+\frac{1}{\Gamma(\beta)} \int_{0}^{T}(T-s)^{\beta-1} \eta(s, y(s)) d s, & t \in\left[0, s_{0}\right], \\ I_{s_{k-1}, t_{k}}^{\beta}\left(\xi_{k}(t)\right), & t \in\left(s_{k-1}, t_{k}\right], \\ \frac{1}{\Gamma(\beta)} \int_{0}^{t}(t-s)^{\beta-1} f(s) d s+\frac{1}{\Gamma(\beta)} \int_{s_{k-1}}^{t_{k}}\left(t_{k}-s\right)^{\beta-1} \xi_{k}(s) d s & k=1,2, \ldots, m, \\ \quad-\frac{1}{\Gamma(\beta)} \int_{0}^{t_{k}}\left(t_{k}-s\right)^{\beta-1} f(s) d s, & t \in\left(t_{k}, s_{k}\right], \\ & k=1,2, \ldots, m .\end{cases}
$$

Proof For $t \in\left[0, s_{0}\right]$, consider

$$
{ }^{c} D_{0, t}^{\beta} y(t)=f(t), \quad y(0)=I_{0, T}^{\beta} \eta(t, y(t)) .
$$

Using Lemma 2.5 and the initial condition, we can get

$$
y(t)=\frac{1}{\Gamma(\beta)} \int_{0}^{t}(t-s)^{\beta-1} f(s) d s+\frac{1}{\Gamma(\beta)} \int_{0}^{T}(T-s)^{\beta-1} \eta(s, y(s)) d s, \quad t \in\left[0, s_{0}\right] .
$$

If $t \in\left(s_{0}, t_{1}\right]$, then $y(t)=I_{s_{0}, t_{1}}^{\beta} \xi_{1}(t)$. For $t \in\left(t_{1}, s_{1}\right]$, we consider

$$
{ }^{c} D_{0, t}^{\beta} y(t)=f(t), \quad y\left(t_{1}\right)=I_{s_{0}, t_{1}}^{\beta} \xi_{1}\left(t_{1}\right) .
$$


Applying Lemma 2.5 to the above equation and using the condition $y\left(t_{1}\right)=I_{s_{0}, t_{1}}^{\beta} \xi_{1}\left(t_{1}\right)$, we obtain

$$
y(t)=\frac{1}{\Gamma(\beta)} \int_{0}^{t}(t-s)^{\beta-1} f(s) d s+\frac{1}{\Gamma(\beta)} \int_{s_{0}}^{t_{1}}\left(t_{1}-s\right)^{\beta-1} \xi_{1}(s) d s-\frac{1}{\Gamma(\beta)} \int_{0}^{t_{1}}\left(t_{1}-s\right)^{\beta-1} f(s) d s .
$$

If $t \in\left(s_{1}, t_{2}\right]$, then $y(t)=I_{s_{1}, t_{2}}^{\beta} \xi_{2}(t)$. When $t \in\left(t_{2}, s_{2}\right]$, we consider

$$
{ }^{c} D_{0, t}^{\beta} y(t)=f(t), \quad y\left(t_{2}\right)=I_{s_{1}, t_{2}}^{\beta} \xi_{2}\left(t_{2}\right) .
$$

With the help of Lemma 2.5 and condition $y\left(t_{2}\right)=I_{s_{1}, t_{2}}^{\beta} \xi_{2}\left(t_{2}\right)$, we get

$$
y(t)=\frac{1}{\Gamma(\beta)} \int_{0}^{t}(t-s)^{\beta-1} f(s) d s+\frac{1}{\Gamma(\beta)} \int_{s_{1}}^{t_{2}}\left(t_{2}-s\right)^{\beta-1} \xi_{2}(s) d s-\frac{1}{\Gamma(\beta)} \int_{0}^{t_{2}}\left(t_{2}-s\right)^{\beta-1} f(s) d s .
$$

Generally, if $t \in\left(s_{k-1}, t_{k}\right]$, then $y(t)=I_{s_{k-1}, t_{k}}^{\beta} \xi_{k}(t)$. For $t \in\left(t_{k}, s_{k}\right]$, we consider

$$
{ }^{c} D_{0, t}^{\beta} y(t)=f(t), \quad y\left(t_{k}\right)=I_{s_{k-1}, t_{k}}^{\beta} \xi_{k}\left(t_{k}\right)
$$

Using Lemma 2.5 and condition $y\left(t_{k}\right)=I_{s_{k-1}, t_{k}}^{\beta} \xi_{k}\left(t_{k}\right)$, we obtain

$$
y(t)=\frac{1}{\Gamma(\beta)} \int_{0}^{t}(t-s)^{\beta-1} f(s) d s+\frac{1}{\Gamma(\beta)} \int_{s_{k-1}}^{t_{k}}\left(t_{k}-s\right)^{\beta-1} \xi_{k}(s) d s-\frac{1}{\Gamma(\beta)} \int_{0}^{t_{k}}\left(t_{k}-s\right)^{\beta-1} f(s) d s .
$$

Conversely, let $y \in \hat{\mathfrak{B}}$ be a solution of the impulsive fractional integral equation (3.2). Using the fact that ${ }^{c} D_{0, t}^{\beta}$ is the left inverse of $I_{0, t}^{\beta}$, we can easily get our required result.

Remark 3.2 If $x \in \hat{\mathfrak{B}}$ represents a solution of inequality (2.1), then $x \in \hat{\mathfrak{B}}$ is a solution of the following integral inequality:

$$
\left\{\begin{array}{rlrl}
\mid x(t) & -\frac{1}{\Gamma(\beta)} \int_{0}^{t}(t-s)^{\beta-1} f\left(s, x(s),{ }^{c} D_{0, t}^{\beta} x(s)\right) d s & & \\
& \quad-\frac{1}{\Gamma(\beta)} \int_{0}^{T}(T-s)^{\beta-1} \eta(s, x(s)) d s \mid & & \\
\leq & \frac{\varepsilon t^{\beta}}{\Gamma(\beta+1)}, & & t \in\left[0, s_{0}\right], \\
\mid x(t) & -I_{s_{k-1}, t_{k}}^{\beta} \xi_{k}(t, x(t)) \mid \leq \varepsilon, & & t \in\left(s_{k-1}, t_{k}\right], k=1,2, \ldots, m, \\
\mid x(t) & -\frac{1}{\Gamma(\beta)} \int_{0}^{t}(t-s)^{\beta-1} f\left(s, x(s),{ }^{c} D_{0, t}^{\beta} x(s)\right) d s & \\
\quad-\frac{1}{\Gamma(\beta)} \int_{s_{k-1}}^{t_{k}}\left(t_{k}-s\right)^{\beta-1} \xi_{k}(s, x(s)) d s & \\
\quad+\frac{1}{\Gamma(\beta)} \int_{0}^{t_{k}}\left(t_{k}-s\right)^{\beta-1} f\left(s, x(s),{ }^{c} D_{0, t}^{\beta} x(s)\right) d s \mid & \\
\leq \frac{\varepsilon}{\Gamma(\beta+1)}\left\{t^{\beta}+\left(t_{k}-s_{k-1}\right)^{\beta}+t_{k}^{\beta}\right\}, & & t \in\left(t_{k}, s_{k}\right], k=1,2, \ldots, m .
\end{array}\right.
$$

As from Remark 2.11, we have

$$
\begin{cases}{ }^{C} D_{0, t}^{\beta} x(t)=f\left(t, x(t),{ }^{C} D_{0, t}^{\beta} x(t)\right)+Q(t), & t \in\left(t_{k}, s_{k}\right], k=0,1, \ldots, m, \\ x(t)=I_{s_{k-1}, t_{k}}^{\beta}\left(\xi_{k}(t, x(t))\right)+Q_{k}, & t \in\left(s_{k-1}, t_{k}\right], k=1,2, \ldots, m, \\ x(0)=I_{0, T}^{\beta} \eta(t, x(t)) . & \end{cases}
$$


Using Lemma 3.1, the solution of the above problem is

$$
x(t)= \begin{cases}\frac{1}{\Gamma(\beta)} \int_{0}^{t}(t-s)^{\beta-1}\left[f\left(s, x(s),{ }^{c} D_{0, t}^{\beta} x(s)\right)+Q(s)\right] d s & \\ \quad+\frac{1}{\Gamma(\beta)} \int_{0}^{T}(T-s)^{\beta-1} \eta(s, x(s)) d s, & t \in\left[0, s_{0}\right], \\ I_{s_{k-1}, t_{k}}^{\beta}\left(\xi_{k}(t, x(t))\right)+Q_{k}, & t \in\left(s_{k-1}, t_{k}\right], \\ \frac{1}{\Gamma(\beta)} \int_{0}^{t}(t-s)^{\beta-1}\left[f\left(s, x(s),{ }^{c} D_{0, t}^{\beta} x(s)\right)+Q(s)\right] d s & \\ \quad+\frac{1}{\Gamma(\beta)} \int_{s_{k-1}}^{t_{k}}\left(t_{k}-s\right)^{\beta-1}\left[\xi_{k}(s, x(s))+Q_{k}\right] d s & \\ -\frac{1}{\Gamma(\beta)} \int_{0}^{t_{k}}\left(t_{k}-s\right)^{\beta-1}\left[f\left(s, x(s),{ }^{c} D_{0, t}^{\beta} x(s)\right)+Q(s)\right] d s, & t \in\left(t_{k}, s_{k}\right], \\ & k=1,2, \ldots, m .\end{cases}
$$

With the help of the above solution and Remark 2.11, we verified Remark 3.2 in the following lines.

For $t \in\left[0, s_{0}\right]$, we consider

$$
\begin{aligned}
\mid x(t) & -\frac{1}{\Gamma(\beta)} \int_{0}^{t}(t-s)^{\beta-1} f\left(s, x(s),{ }^{c} D_{0, t}^{\beta} x(s)\right) d s-\frac{1}{\Gamma(\beta)} \int_{0}^{T}(T-s)^{\beta-1} \eta(s, x(s)) d s \mid \\
= & \mid \frac{1}{\Gamma(\beta)} \int_{0}^{t}(t-s)^{\beta-1}\left[f\left(s, x(s),{ }^{c} D_{0, t}^{\beta} x(s)\right)+Q(s)\right] d s+\frac{1}{\Gamma(\beta)} \int_{0}^{T}(T-s)^{\beta-1} \eta(s, x(s)) d s \\
& -\frac{1}{\Gamma(\beta)} \int_{0}^{t}(t-s)^{\beta-1} f\left(s, x(s),{ }^{c} D_{0, t}^{\beta} x(s)\right) d s-\frac{1}{\Gamma(\beta)} \int_{0}^{T}(T-s)^{\beta-1} \eta(s, x(s)) d s \mid \\
\leq & \frac{1}{\Gamma(\beta)} \int_{0}^{t}(t-s)^{\beta-1}|Q(s)| d s \\
\leq & \frac{\varepsilon}{\Gamma(\beta)} \int_{0}^{t}(t-s)^{\beta-1} d s \\
\leq & \frac{\varepsilon t^{\beta}}{\Gamma(\beta+1)} .
\end{aligned}
$$

For $t \in\left(s_{k-1}, t_{k}\right], k=1,2, \ldots, m$, consider

$$
\begin{aligned}
\left|x(t)-I_{s_{k-1}, t_{k}}^{\beta} \xi_{k}(t, x(t))\right| & =\left|I_{s_{k-1}, t_{k}}^{\beta} \xi_{k}(t, x(t))+Q_{k}-I_{s_{k-1}, t_{k}}^{\beta} \xi_{k}(t, x(t))\right| \\
& =\left|Q_{k}\right| \leq \varepsilon, \quad k=1,2, \ldots, m .
\end{aligned}
$$

For $t \in\left(t_{k}, s_{k}\right], k=1,2, \ldots, m$, we consider

$$
\begin{aligned}
\mid x(t) & -\frac{1}{\Gamma(\beta)} \int_{0}^{t}(t-s)^{\beta-1} f\left(s, x(s),{ }^{c} D_{0, t}^{\beta} x(s)\right) d s-\frac{1}{\Gamma(\beta)} \int_{s_{k-1}}^{t_{k}}\left(t_{k}-s\right)^{\beta-1} \xi_{k}(s, x(s)) d s \\
& +\frac{1}{\Gamma(\beta)} \int_{0}^{t_{k}}\left(t_{k}-s\right)^{\beta-1} f\left(s, x(s),{ }^{c} D_{0, t}^{\beta} x(s)\right) d s \mid \\
= & \frac{1}{\Gamma(\beta)} \int_{0}^{t}(t-s)^{\beta-1}\left[f\left(s, x(s),{ }^{c} D_{0, t}^{\beta} x(s)\right)+Q(s)\right] d s \\
& +\frac{1}{\Gamma(\beta)} \int_{s_{k-1}}^{t_{k}}\left(t_{k}-s\right)^{\beta-1}\left[\xi_{k}(s, x(s))+Q_{k}\right] d s \\
& -\frac{1}{\Gamma(\beta)} \int_{0}^{t_{k}}\left(t_{k}-s\right)^{\beta-1}\left[f\left(s, x(s),{ }^{c} D_{0, t}^{\beta} x(s)\right)+Q(s)\right] d s
\end{aligned}
$$




$$
\begin{aligned}
& -\frac{1}{\Gamma(\beta)} \int_{0}^{t}(t-s)^{\beta-1} f\left(s, x(s),{ }^{c} D_{0, t}^{\beta} x(s)\right) d s \\
& -\frac{1}{\Gamma(\beta)} \int_{s_{k-1}}^{t_{k}}\left(t_{k}-s\right)^{\beta-1} \xi_{k}(s, x(s)) d s+\frac{1}{\Gamma(\beta)} \int_{0}^{t_{k}}\left(t_{k}-s\right)^{\beta-1} f\left(s, x(s),{ }^{c} D_{0, t}^{\beta} x(s)\right) d s \mid \\
\leq & \frac{1}{\Gamma(\beta)} \int_{0}^{t}(t-s)^{\beta-1}|Q(s)| d s+\frac{1}{\Gamma(\beta)} \int_{s_{k-1}}^{t_{k}}\left(t_{k}-s\right)^{\beta-1}\left|Q_{k}\right| d s \\
& +\frac{1}{\Gamma(\beta)} \int_{0}^{t_{k}}\left(t_{k}-s\right)^{\beta-1}|Q(s)| d s \\
\leq & \frac{\varepsilon}{\Gamma(\beta)} \int_{0}^{t}(t-s)^{\beta-1} d s+\frac{\varepsilon}{\Gamma(\beta)} \int_{s_{k-1}}^{t_{k}}\left(t_{k}-s\right)^{\beta-1} d s+\frac{\varepsilon}{\Gamma(\beta)} \int_{0}^{t_{k}}\left(t_{k}-s\right)^{\beta-1} d s \\
\leq & \frac{\varepsilon t^{\beta}}{\beta \Gamma(\beta)}+\frac{\varepsilon\left(t_{k}-s_{k-1}\right)^{\beta}}{\beta \Gamma(\beta)}+\frac{\varepsilon t_{k}^{\beta}}{\beta \Gamma(\beta)} \\
= & \frac{\varepsilon}{\Gamma(\beta+1)}\left\{t^{\beta}+\left(t_{k}-s_{k-1}\right)^{\beta}+t_{k}^{\beta}\right\} .
\end{aligned}
$$

Hence Remark 3.2 is verified.

Remark 3.3 If $x \in \hat{\mathfrak{B}}$ represents a solution of inequality (2.2), then $x \in \hat{\mathfrak{B}}$ is a solution of the following integral inequality:

$$
\left\{\begin{aligned}
\mid x(t) & -\frac{1}{\Gamma(\beta)} \int_{0}^{t}(t-s)^{\beta-1} f\left(s, x(s),{ }^{c} D_{0, t}^{\beta} x(s)\right) d s & & \\
& -\frac{1}{\Gamma(\beta)} \int_{0}^{T}(T-s)^{\beta-1} \eta(s, x(s)) d s \mid & & \\
\leq & \frac{1}{\Gamma(\beta)} \int_{0}^{t}(t-s)^{\beta-1} \phi(s) d s, & & t \in\left[0, s_{0}\right], \\
\mid x(t) & -I_{s_{k-1}, t_{k}}^{\beta} \xi_{k}(t, x(t)) \mid \leq \psi, & & t \in\left(s_{k-1}, t_{k}\right], k=1,2, \ldots, m, \\
\mid x(t) & -\frac{1}{\Gamma(\beta)} \int_{0}^{t}(t-s)^{\beta-1} f\left(s, x(s),{ }^{c} D_{0, t}^{\beta} x(s)\right) d s & & \\
& -\frac{1}{\Gamma(\beta)} \int_{s_{k-1}}^{t_{k}}\left(t_{k}-s\right)^{\beta-1} \xi_{k}(s, x(s)) d s & & \\
& +\frac{1}{\Gamma(\beta)} \int_{0}^{t_{k}}\left(t_{k}-s\right)^{\beta-1} f\left(s, x(s),{ }^{c} D_{0, t}^{\beta} x(s)\right) d s \mid & & \\
\leq & \frac{1}{\Gamma(\beta)} \int_{0}^{t}(t-s)^{\beta-1} \phi(s) d s & & \\
& +\frac{1}{\Gamma(\beta)} \int_{0}^{t_{k}}\left(t_{k}-s\right)^{\beta-1} \phi(s) d s+\frac{\psi\left(t_{k}-s_{k-1}\right)^{\beta}}{\Gamma(\beta+1)}, & & t \in\left(t_{k}, s_{k}\right], k=1,2, \ldots, m .
\end{aligned}\right.
$$

Remark 3.4 If $x \in \hat{\mathfrak{B}}$ represents a solution of inequality (2.3), then $x \in \hat{\mathfrak{B}}$ is a solution of the following integral inequality:

$$
\left\{\begin{array}{rlrl}
\mid x(t) & -\frac{1}{\Gamma(\beta)} \int_{0}^{t}(t-s)^{\beta-1} f\left(s, x(s),{ }^{c} D_{0, t}^{\beta} x(s)\right) d s & \\
\quad-\frac{1}{\Gamma(\beta)} \int_{0}^{T}(T-s)^{\beta-1} \eta(s, x(s)) d s \mid & \\
\leq \frac{\varepsilon}{\Gamma(\beta)} \int_{0}^{t}(t-s)^{\beta-1} \phi(s) d s, & & t \in\left[0, s_{0}\right], \\
\mid x(t) & -I_{s_{k-1}, t_{k}}^{\beta} \xi_{k}(t, x(t)) \mid \leq \varepsilon \psi, & & t \in\left(s_{k-1}, t_{k}\right], k=1,2, \ldots, m, \\
\mid x(t) & -\frac{1}{\Gamma(\beta)} \int_{0}^{t}(t-s)^{\beta-1} f\left(s, x(s),{ }^{c} D_{0, t}^{\beta} x(s)\right) d s & \\
\quad-\frac{1}{\Gamma(\beta)} \int_{s_{k-1}}^{t_{k}}\left(t_{k}-s\right)^{\beta-1} \xi_{k}(s, x(s)) d s & \\
\quad+\frac{1}{\Gamma(\beta)} \int_{0}^{t_{k}}\left(t_{k}-s\right)^{\beta-1} f\left(s, x(s),{ }^{c} D_{0, t}^{\beta} x(s)\right) d s \mid & \\
\leq & \frac{\varepsilon}{\Gamma(\beta)} \int_{0}^{t}(t-s)^{\beta-1} \phi(s) d s & \\
\quad+\frac{\varepsilon}{\Gamma(\beta)} \int_{0}^{t_{k}}\left(t_{k}-s\right)^{\beta-1} \phi(s) d s+\frac{\varepsilon \psi\left(t_{k}-s_{k-1}\right)^{\beta}}{\Gamma(\beta+1)}, & & t \in\left(t_{k}, s_{k}\right], k=1,2, \ldots, m .
\end{array}\right.
$$

By using Remark 2.12 and Remark 2.13, we can verify Remark 3.3 and Remark 3.4, respectively, by the same procedure as we used for verification of Remark 3.2. 


\section{Main results}

In this section, we prove four different types of Ulam stability results for problem (1.3). We also establish some conditions for the existence and uniqueness of the solutions of problem (1.3) in view of Theorem 2.16.

First, we introduce the following assumptions:

$\left(A_{1}\right)$ The function $f: J \times \mathbb{R} \times \mathbb{R} \rightarrow \mathbb{R}$ is continuous.

$\left(A_{2}\right)$ There exist constants $\bar{K}_{f}>0$ and $0<\bar{L}_{f}<1$ such that

$$
\left|f\left(t, \omega_{1}, \varpi_{1}\right)-f\left(t, \omega_{2}, \varpi_{2}\right)\right| \leq \bar{K}_{f}\left|\omega_{1}-\omega_{2}\right|+\bar{L}_{f}\left|\varpi_{1}-\varpi_{2}\right|
$$

for all $\omega_{1}, \omega_{2}, \varpi_{1}, \varpi_{2} \in \mathbb{R}$ and $t \in J$.

$\left(A_{3}\right)$ There exists a constant $L_{\eta}>0$ such that

$$
|\eta(t, \omega)-\eta(t, \varpi)| \leq L_{\eta}|\omega-\varpi|
$$

for all $\omega, \varpi \in \mathbb{R}$ and $t \in J$.

$\left(A_{4}\right)$ There exists a constant $L_{\xi_{k}}>0$ such that

$$
\left|I_{s_{k-1}, t_{k}}^{\beta} \xi(t, \omega)-I_{s_{k-1}, t_{k}}^{\beta} \xi(t, \varpi)\right| \leq L_{\xi_{k}}|\omega-\varpi|
$$

for all $\omega, \varpi \in \mathbb{R}$ and $t \in\left(s_{k-1}, t_{k}\right], k=1,2, \ldots, m$.

$\left(A_{5}\right)$ Let $\phi: J \rightarrow \mathbb{R}_{+}$be any continuous nondecreasing function from the space of continuous functions $C\left(J, \mathbb{R}_{+}\right)$, there exist nonnegative constants $c_{\phi}$ and $c_{\beta}$ such that

$$
\left(\int_{0}^{t}(\phi(s))^{\frac{1}{\gamma}} d s\right)^{\gamma} \leq c_{\phi} \phi(t), \quad t \in J
$$

and

$$
\left(\int_{0}^{t}\left({ }^{c} D_{0, t}^{\beta} \phi(s)\right)^{\frac{1}{\gamma}} d s\right)^{\gamma} \leq c_{\beta} \phi(t), \quad t \in J
$$

where $0<\gamma<\beta \leq 1$.

Theorem 4.1 Assume that $\left(A_{1}\right)-\left(A_{5}\right)$ are true and a function $x \in \hat{\mathfrak{B}}$ satisfies inequality (2.3), then there exists a unique solution $x_{0}$ of problem (1.3) such that

$$
x_{0}(t)= \begin{cases}\frac{1}{\Gamma(\beta)} \int_{0}^{t}(t-s)^{\beta-1} f\left(s, x_{0}(s),{ }^{c} D_{0, t}^{\beta} x_{0}(s)\right) d s & \\ \quad+\frac{1}{\Gamma(\beta)} \int_{0}^{T}(T-s)^{\beta-1} \eta\left(s, x_{0}(s)\right) d s, & t \in\left[0, s_{0}\right], \\ I_{s_{k-1}, t_{k}}^{\beta}\left(\xi_{k}\left(t, x_{0}(t)\right)\right), & t \in\left(s_{k-1}, t_{k}\right], \\ \frac{1}{\Gamma(\beta)} \int_{0}^{t}(t-s)^{\beta-1} f\left(s, x_{0}(s),{ }^{c} D_{0, t}^{\beta} x_{0}(s)\right) d s & \\ \quad+\frac{1}{\Gamma(\beta)} \int_{s_{k-1}}^{t_{k}}\left(t_{k}-s\right)^{\beta-1} \xi_{k}\left(s, x_{0}(s)\right) d s, \ldots, m, \\ \quad-\frac{1}{\Gamma(\beta)} \int_{0}^{t_{k}}\left(t_{k}-s\right)^{\beta-1} f\left(s, x_{0}(s),{ }^{c} D_{0, t}^{\beta} x_{0}(s)\right) d s, & t \in\left(t_{k}, s_{k}\right], \\ & k=1,2, \ldots, m,\end{cases}
$$

and

$$
\left|x(t)-x_{0}(t)\right| \leq \frac{\left[\frac{c_{\phi}}{\Gamma(\beta)}\left(\frac{1-\gamma}{\beta-\gamma}\right)^{\beta-\gamma}\left(T^{\beta-\gamma}+t_{k}^{\beta-\gamma}\right)+\frac{\left(t_{k}-s_{k-1}\right)^{\beta}}{\Gamma(\beta+1)}\right] \varepsilon(\phi(t)+\psi)}{1-L}
$$


for all $t \in J, 0<\gamma<\beta \leq 1, t_{k}, s_{k-1}$ are considered to be maximum values and $L=$ $\max \left\{L_{1}, L_{2}\right\}<1$, where

$$
\begin{aligned}
& L_{1}=\max \left\{\frac{\bar{K}_{f} c_{\phi}+\bar{L}_{f} c_{\beta}}{\Gamma(\beta)}\left(\frac{1-\gamma}{\beta-\gamma}\right)^{1-\gamma}\left(s_{k}^{\beta-\gamma}+t_{k}^{\beta-\gamma}\right)+L_{\xi_{k}} \mid k=0,1, \ldots, m\right\}, \\
& L_{2}=\max \left\{\frac{\bar{K}_{f}}{\Gamma(\beta+1)}\left(s_{k}^{\beta}+t_{k}^{\beta}\right)+L_{\xi_{k}} \mid k=1,2, \ldots, m\right\},
\end{aligned}
$$

i.e., problem (1.3) is Ulam-Hyers-Rassias stable.

Proof Let $Y$ be the space of piecewise continuous functions defined by

$$
Y=\{p: J \rightarrow \mathbb{R} \mid p \in \hat{\mathfrak{B}}\}
$$

and let us introduce a generalized complete metric on $Y$ as

$$
d(p, q)=\inf \left\{C_{1}+C_{2} \in[0, \infty]|| p(t)-q(t) \mid \leq\left(C_{1}+C_{2}\right) \varepsilon(\phi(t)+\psi)\right\},
$$

for all $t \in J$, where

$$
C_{1} \in\{C \in[0, \infty]|| p(t)-q(t) \mid \leq C \varepsilon \phi(t)\}, \quad t \in\left(t_{k}, s_{k}\right], k=0,1, \ldots, m
$$

and

$$
C_{2} \in\{C \in[0, \infty]|| p(t)-q(t) \mid \leq C \varepsilon \psi\}, \quad t \in\left(s_{k-1}, t_{k}\right], k=, 1, \ldots, m
$$

Now, we define an operator $\Lambda: Y \rightarrow Y$ by

$$
(\Lambda y)(t)= \begin{cases}\frac{1}{\Gamma(\beta)} \int_{0}^{t}(t-s)^{\beta-1} f\left(s, y(s),{ }^{c} D_{0, t}^{\beta} y(s)\right) d s & \\ \quad+\frac{1}{\Gamma(\beta)} \int_{0}^{T}(T-s)^{\beta-1} \eta(s, y(s)) d s, & t \in\left[0, s_{0}\right], \\ I_{s_{k-1}, t_{k}}^{\beta}\left(\xi_{k}(t, y(t))\right), & t \in\left(s_{k-1}, t_{k}\right], \\ \frac{1}{\Gamma(\beta)} \int_{0}^{t}(t-s)^{\beta-1} f\left(s, y(s),{ }^{c} D_{0, t}^{\beta} y(s)\right) d s & \\ \quad+\frac{1}{\Gamma(\beta)} \int_{s_{k-1}}^{t_{k}}\left(t_{k}-s\right)^{\beta-1} \xi_{k}(s, y(s)) d s, \ldots, m, \\ \quad-\frac{1}{\Gamma(\beta)} \int_{0}^{t_{k}}\left(t_{k}-s\right)^{\beta-1} f\left(s, y(s),{ }^{c} D_{0, t}^{\beta} y(s)\right) d s, & t \in\left(t_{k}, s_{k}\right], \\ & k=1,2, \ldots, m,\end{cases}
$$

for all $y \in Y$. Clearly, $\Lambda$ is a well-defined operator because $y$ and $f$ are continuous functions.

To achieve our goal, first we need to prove that $\Lambda$ is strictly contractive on $Y$. For this we take any $p, q \in Y$ and $C_{1}, C_{2} \in[0, \infty]$, using Eq. (4.4), we get

$$
|p(t)-q(t)| \leq \begin{cases}C_{1} \varepsilon \phi(t), & t \in\left(t_{k}, s_{k}\right], k=0,1, \ldots, m, \\ C_{2} \varepsilon \psi, & t \in\left(s_{k-1}, t_{k}\right], k=1,2, \ldots, m\end{cases}
$$

From the linearity property of Caputo fractional derivative, Eqs. (4.5), (4.6) and assumptions $\left(A_{2}\right)-\left(A_{5}\right)$, we obtain the following. 
Case 1: For $t \in\left[0, s_{0}\right]$, we have

$$
\begin{aligned}
& |(\Lambda p)(t)-(\Lambda q)(t)|=\mid \frac{1}{\Gamma(\beta)} \int_{0}^{t}(t-s)^{\beta-1} f\left(s, p(s),{ }^{c} D_{0, t}^{\beta} p(s)\right) d s \\
& +\frac{1}{\Gamma(\beta)} \int_{0}^{T}(T-s)^{\beta-1} \eta(s, p(s)) d s \\
& -\frac{1}{\Gamma(\beta)} \int_{0}^{t}(t-s)^{\beta-1} f\left(s, q(s),{ }^{c} D_{0, t}^{\beta} q(s)\right) d s \\
& -\frac{1}{\Gamma(\beta)} \int_{0}^{T}(T-s)^{\beta-1} \eta(s, q(s)) d s \mid \\
& \leq \frac{1}{\Gamma(\beta)} \int_{0}^{t}(t-s)^{\beta-1}\left|f\left(s, p(s),{ }^{c} D_{0, t}^{\beta} p(s)\right)-f\left(s, q(s),{ }^{c} D_{0, t}^{\beta} q(s)\right)\right| d s \\
& +\frac{1}{\Gamma(\beta)} \int_{0}^{T}(T-s)^{\beta-1}|\eta(s, p(s))-\eta(s, q(s))| d s \\
& \leq \frac{1}{\Gamma(\beta)} \int_{0}^{t}(t-s)^{\beta-1}\left[\bar{K}_{f}|p(s)-q(s)|+{\overline{L_{k}}}^{c}{ }^{c} D_{0, t}^{\beta} p(s)-{ }^{c} D_{0, t}^{\beta} q(s) \mid\right] d s \\
& +\frac{1}{\Gamma(\beta)} \int_{0}^{T}(T-s)^{\beta-1}\left[L_{\eta}|p(s)-q(s)|\right] d s \\
& \leq \frac{\bar{K}_{f} C_{1} \varepsilon}{\Gamma(\beta)} \int_{0}^{t}(t-s)^{\beta-1} \phi(s) d s+\frac{\overline{L_{f}} C_{1} \varepsilon}{\Gamma(\beta)} \int_{0}^{t}(t-s)^{\beta-1}\left({ }^{c} D_{0, t}^{\beta} \phi(s)\right) d s \\
& +\frac{\overline{L_{\eta}} C_{1} \varepsilon}{\Gamma(\beta)} \int_{0}^{T}(T-s)^{\beta-1} \phi(s) d s \\
& \leq \frac{\bar{K}_{f} C_{1} \varepsilon}{\Gamma(\beta)}\left(\int_{0}^{t}(t-s)^{\frac{\beta-1}{1-\gamma}} d s\right)^{1-\gamma}\left(\int_{0}^{t}(\phi(s))^{\frac{1}{\gamma}} d s\right)^{\gamma} \\
& +\frac{\overline{L_{f}} C_{1} \varepsilon}{\Gamma(\beta)}\left(\int_{0}^{t}(t-s)^{\frac{\beta-1}{1-\gamma}} d s\right)^{1-\gamma}\left(\int_{0}^{t}\left({ }^{c} D_{0, t}^{\beta} \phi(s)\right)^{\frac{1}{\gamma}} d s\right)^{\gamma} \\
& +\frac{L_{\eta} C_{1} \varepsilon}{\Gamma(\beta)}\left(\int_{0}^{T}(T-s)^{\frac{\beta-1}{1-\gamma}} d s\right)^{1-\gamma}\left(\int_{0}^{T}(\phi(s))^{\frac{1}{\gamma}} d s\right)^{\gamma} \\
& \leq \frac{\bar{K}_{f} C_{1} \varepsilon}{\Gamma(\beta)}\left(\frac{1-\gamma}{\beta-\gamma}\right)^{1-\gamma} t^{\beta-\gamma} c_{\phi} \phi(t)+\frac{\overline{L_{f}} C_{1} \varepsilon}{\Gamma(\beta)}\left(\frac{1-\gamma}{\beta-\gamma}\right)^{1-\gamma} t^{\beta-\gamma} c_{\beta} \phi(t) \\
& +\frac{L_{\eta} C_{1} \varepsilon}{\Gamma(\beta)}\left(\frac{1-\gamma}{\beta-\gamma}\right)^{1-\gamma} T^{\beta-\gamma} c_{\phi} \phi(t) \\
& \leq\left[\frac{\bar{K}_{f} c_{\phi}\left(\frac{1-\gamma}{\beta-\gamma}\right)^{1-\gamma}}{\Gamma(\beta)} s_{0}^{\beta-\gamma}+\frac{\bar{L}_{f} c_{\beta}\left(\frac{1-\gamma}{\beta-\gamma}\right)^{1-\gamma}}{\Gamma(\beta)} s_{0}^{\beta-\gamma}+\frac{L_{\eta} c_{\phi}\left(\frac{1-\gamma}{\beta-\gamma}\right)^{1-\gamma}}{\Gamma(\beta)} T^{\beta-\gamma}\right] \\
& \times C_{1} \varepsilon \phi(t) \\
& =\frac{\left(\frac{1-\gamma}{\beta-\gamma}\right)^{1-\gamma}}{\Gamma(\beta)}\left[\left(\bar{K}_{f} c_{\phi}+\bar{L}_{f} c_{\beta}\right) s_{0}^{\beta-\gamma}+L_{\eta} c_{\phi} T^{\beta-\gamma}\right] C_{1} \varepsilon \phi(t) .
\end{aligned}
$$

Case 2: For $t \in\left(s_{k-1}, t_{k}\right], k=1,2, \ldots, m$, we have

$$
\begin{aligned}
|(\Lambda p)(t)-(\Lambda q)(t)| & =\left|I_{s_{k-1}, t_{k}}^{\beta} \xi_{k}(t, p(t))-I_{s_{k-1}, t_{k}}^{\beta} \xi_{k}(t, q(t))\right| \\
& \leq L_{\xi_{k}}|p(t)-q(t)| \leq L_{\xi_{k}} C_{2} \varepsilon \psi .
\end{aligned}
$$


Zada et al. Advances in Difference Equations ( 2017) 2017:317

Page 13 of 26

Case 3(a): For $t \in\left(t_{k}, s_{k}\right]$ and $s \in\left(t_{k}, s_{k}\right], k=1,2, \ldots, m$, we have

$$
\begin{aligned}
& |(\Lambda p)(t)-(\Lambda q)(t)|=\mid \frac{1}{\Gamma(\beta)} \int_{0}^{t}(t-s)^{\beta-1} f\left(s, p(s),{ }^{c} D_{0, t}^{\beta} p(s)\right) d s \\
& +\frac{1}{\Gamma(\beta)} \int_{s_{k-1}}^{t_{k}}\left(t_{k}-s\right)^{\beta-1} \xi_{k}(s, p(s)) d s \\
& -\frac{1}{\Gamma(\beta)} \int_{0}^{t_{k}}\left(t_{k}-s\right){ }^{\beta-1} f\left(s, p(s),{ }^{c} D_{0, t}^{\beta} p(s)\right) d s \\
& -\frac{1}{\Gamma(\beta)} \int_{0}^{t}(t-s)^{\beta-1} f\left(s, q(s),{ }^{c} D_{0, t}^{\beta} q(s)\right) d s \\
& -\frac{1}{\Gamma(\beta)} \int_{s_{k-1}}^{t_{k}}\left(t_{k}-s\right)^{\beta-1} \xi_{k}(s, q(s)) d s \\
& +\frac{1}{\Gamma(\beta)} \int_{0}^{t_{k}}\left(t_{k}-s\right)^{\beta-1} f\left(s, q(s),{ }^{c} D_{0, t}^{\beta} q(s)\right) d s \mid \\
& \leq \mid \frac{1}{\Gamma(\beta)} \int_{0}^{t}(t-s)^{\beta-1} f\left(s, p(s),{ }^{c} D_{0, t}^{\beta} p(s)\right) d s \\
& -\frac{1}{\Gamma(\beta)} \int_{0}^{t}(t-s)^{\beta-1} f\left(s, q(s),{ }^{c} D_{0, t}^{\beta} q(s)\right) d s \mid \\
& +\mid \frac{1}{\Gamma(\beta)} \int_{s_{k-1}}^{t_{k}}\left(t_{k}-s\right)^{\beta-1} \xi_{k}(s, p(s)) d s \\
& -\frac{1}{\Gamma(\beta)} \int_{s_{k-1}}^{t_{k}}\left(t_{k}-s\right)^{\beta-1} \xi_{k}(s, q(s)) d s \mid \\
& +\mid \frac{1}{\Gamma(\beta)} \int_{0}^{t_{k}}\left(t_{k}-s\right)^{\beta-1} f\left(s, q(s),{ }^{c} D_{0, t}^{\beta} q(s)\right) d s \\
& -\frac{1}{\Gamma(\beta)} \int_{0}^{t_{k}}\left(t_{k}-s\right)^{\beta-1} f\left(s, p(s),{ }^{c} D_{0, t}^{\beta} p(s)\right) d s \mid \\
& \leq \frac{\left(\frac{1-\gamma}{\beta-\gamma}\right)^{1-\gamma}}{\Gamma(\beta)}\left[\left(\bar{K}_{f} c_{\phi}+\bar{L}_{f} c_{\beta}\right) s_{k}^{\beta-\gamma}\right] C_{1} \varepsilon \phi(t)+L_{\xi_{k}} C_{2} \varepsilon \psi \\
& +\frac{\left(\frac{1-\gamma}{\beta-\gamma}\right)^{1-\gamma}}{\Gamma(\beta)}\left[\left(\bar{K}_{f} c_{\phi}+\bar{L}_{f} c_{\beta}\right) t_{k}^{\beta-\gamma}\right] C_{1} \varepsilon \phi(t) \\
& \leq\left[\frac{\bar{K}_{f} c_{\phi}+\bar{L}_{f} c_{\beta}}{\Gamma(\beta)}\left(\frac{1-\gamma}{\beta-\gamma}\right)^{1-\gamma}\left(s_{k}^{\beta-\gamma}+t_{k}^{\beta-\gamma}\right)+L_{\xi_{k}}\right] \\
& \times\left(C_{1}+C_{2}\right) \varepsilon(\phi(t)+\psi) .
\end{aligned}
$$

Case 3(b): For $t \in\left(t_{k}, s_{k}\right]$ and $s \in\left(s_{k-1}, t_{k}\right], k=1,2, \ldots, m$, we have

$$
\begin{aligned}
|(\Lambda p)(t)-(\Lambda q)(t)|= & \mid \frac{1}{\Gamma(\beta)} \int_{0}^{t}(t-s)^{\beta-1} f\left(s, p(s),{ }^{c} D_{0, t}^{\beta} p(s)\right) d s \\
& +\frac{1}{\Gamma(\beta)} \int_{s_{k-1}}^{t_{k}}\left(t_{k}-s\right)^{\beta-1} \xi_{k}(s, p(s)) d s \\
& -\frac{1}{\Gamma(\beta)} \int_{0}^{t_{k}}\left(t_{k}-s\right)^{\beta-1} f\left(s, p(s),{ }^{c} D_{0, t}^{\beta} p(s)\right) d s
\end{aligned}
$$




$$
\begin{aligned}
& -\frac{1}{\Gamma(\beta)} \int_{0}^{t}(t-s)^{\beta-1} f\left(s, q(s),{ }^{c} D_{0, t}^{\beta} q(s)\right) d s \\
& -\frac{1}{\Gamma(\beta)} \int_{s_{k-1}}^{t_{k}}\left(t_{k}-s\right)^{\beta-1} \xi_{k}(s, q(s)) d s \\
& +\frac{1}{\Gamma(\beta)} \int_{0}^{t_{k}}\left(t_{k}-s\right)^{\beta-1} f\left(s, q(s),{ }^{c} D_{0, t}^{\beta} q(s)\right) d s \mid \\
\leq & \frac{1}{\Gamma(\beta)} \int_{0}^{t}(t-s)^{\beta-1} f\left(s, p(s),{ }^{c} D_{0, t}^{\beta} p(s)\right) d s \\
& -\frac{1}{\Gamma(\beta)} \int_{0}^{t}(t-s)^{\beta-1} f\left(s, q(s),{ }^{c} D_{0, t}^{\beta} q(s)\right) d s \mid \\
& +\mid \frac{1}{\Gamma(\beta)} \int_{s_{k-1}}^{t_{k}}\left(t_{k}-s\right)^{\beta-1} \xi_{k}(s, p(s)) d s \\
& -\frac{1}{\Gamma(\beta)} \int_{s_{k-1}}^{t_{k}}\left(t_{k}-s\right)^{\beta-1} \xi_{k}(s, q(s)) d s \mid \\
& +\mid \frac{1}{\Gamma(\beta)} \int_{0}^{t_{k}}\left(t_{k}-s\right)^{\beta-1} f\left(s, q(s),{ }^{c} D_{0, t}^{\beta} q(s)\right) d s \\
& -\frac{1}{\Gamma(\beta)} \int_{0}^{t_{k}}\left(t_{k}-s\right)^{\beta-1} f\left(s, p(s),{ }^{c} D_{0, t}^{\beta} p(s)\right) d s \mid \\
\leq & {\left[\frac{\bar{K}_{f}}{\Gamma(\beta+1)}\left(s_{k}^{\beta}+t_{k}^{\beta}\right)+L_{\xi_{k}}\right] C_{2} \varepsilon \psi } \\
\leq & {\left[\frac{\overline{K_{f}}}{\Gamma(\beta+1)}\left(s_{k}^{\beta}+t_{k}^{\beta}\right)+L_{\xi_{k}}\right]\left(C_{1}+C_{2}\right) \varepsilon(\phi(t)+\psi) . }
\end{aligned}
$$

From the above cases, for any $p, q \in Y$ and $L<1$, we obtain

$$
|(\Lambda p)(t)-(\Lambda q)(t)| \leq L\left(C_{1}+C_{2}\right) \varepsilon(\phi(t)+\psi), \quad t \in J,
$$

that is,

$$
d(\Lambda p, \Lambda q) \leq L\left(C_{1}+C_{2}\right) \varepsilon(\phi(t)+\psi), \quad t \in J
$$

which implies that $\Lambda$ is strictly contractive on $Y$.

It follows from Eqs. (4.3) and (4.5) that for any arbitrary $p_{0} \in Y$, there exists a constant $0<\lambda_{1}<\infty$ such that

$$
\begin{aligned}
\left|\left(\Lambda p_{0}\right)(t)-p_{0}(t)\right|= & \mid \frac{1}{\Gamma(\beta)} \int_{0}^{t}(t-s)^{\beta-1} f\left(s, p_{0}(s),{ }^{c} D_{0, t}^{\beta} p_{0}(s)\right) d s \\
& +\frac{1}{\Gamma(\beta)} \int_{0}^{T}(T-s)^{\beta-1} \eta\left(s, p_{0}(s)\right) d s-p_{0}(t) \mid \\
\leq & \lambda_{1} \varepsilon \phi(t) \leq \lambda_{1} \varepsilon(\phi(t)+\psi), \quad t \in\left[0, s_{0}\right],
\end{aligned}
$$

also there is a constant $0<\lambda_{2}<\infty$ such that

$$
\begin{aligned}
\left|\left(\Lambda p_{0}\right)(t)-p_{0}(t)\right| & =\left|I_{s_{k-1}, t_{k}}^{\beta} \xi_{k}\left(t, p_{0}(t)\right)-p_{0}(t)\right| \\
& \leq \lambda_{2} \varepsilon \psi \leq \lambda_{2} \varepsilon(\phi(t)+\psi), \quad t \in\left(s_{k-1}, t_{k}\right], k=1,2, \ldots, m .
\end{aligned}
$$


Furthermore, we can also find a constant $0<\lambda_{3}<\infty$ such that

$$
\begin{aligned}
\left|\left(\Lambda p_{0}\right)(t)-p_{0}(t)\right|= & \mid \frac{1}{\Gamma(\beta)} \int_{0}^{t}(t-s)^{\beta-1} f\left(s, p_{0}(s),{ }^{c} D_{0, t}^{\beta} p_{0}(s)\right) d s \\
& +\frac{1}{\Gamma(\beta)} \int_{s_{k-1}}^{t_{k}}\left(t_{k}-s\right)^{\beta-1} \xi_{k}\left(s, p_{0}(s)\right) d s \\
& -\frac{1}{\Gamma(\beta)} \int_{0}^{t_{k}}\left(t_{k}-s\right)^{\beta-1} f\left(s, p_{0}(s),{ }^{c} D_{0, t}^{\beta} p_{0}(s)\right) d s-p_{0}(t) \mid \\
\leq & \lambda_{3} \varepsilon(\phi(t)+\psi)
\end{aligned}
$$

for all $t \in\left(t_{k}, s_{k}\right], k=1,2, \ldots, m$.

Since $f, \xi_{k}$ and $p_{0}$ are bounded on $J$ and $\phi(\cdot)+\psi>0$, therefore Eq. (4.4) implies that

$$
d\left(\Lambda p_{0}, p_{0}\right)<\infty
$$

By using Theorem 2.16(a), there exists a continuous function $x_{0}: J \rightarrow \mathbb{R}$ such that $\Lambda^{n} p_{0} \rightarrow$ $x_{0}$ in $(Y, d)$ as $n \rightarrow \infty$ and $\Lambda x_{0}=x_{0}$, that is, $x_{0}$ satisfies Eq. (4.1) for all $t \in J$.

Next, we verify that

$$
\left\{p \in Y \mid d\left(p_{0}, p\right)<\infty\right\}=Y
$$

For any $p \in Y$, since $p$ and $p_{0}$ are bounded on $J$ and $\min _{t \in J} \varepsilon(\phi(t)+\psi)>0$, there exists a constant $0<\lambda_{p}<\infty$ such that

$$
\left|p_{0}(t)-p(t)\right| \leq \lambda_{p} \varepsilon(\phi(t)+\psi), \quad t \in J
$$

Therefore, we get $d\left(\Lambda p_{0}, p_{0}\right)<\infty$ for all $p \in Y$, that is,

$$
\left\{p \in Y \mid d\left(p_{0}, p\right)<\infty\right\}=Y
$$

Hence, in view of Theorem 2.16(b), we conclude that $x_{0}$ is the unique continuous function with property (4.1).

On the other hand, it follows from Remark 3.4 and assumption $\left(A_{5}\right)$ that: for $t \in\left[0, s_{0}\right]$, we have

$$
\begin{aligned}
\mid x(t) & -\frac{1}{\Gamma(\beta)} \int_{0}^{t}(t-s)^{\beta-1} f\left(s, x(s),{ }^{c} D_{0, t}^{\beta} x(s)\right) d s \\
& -\frac{1}{\Gamma(\beta)} \int_{0}^{T}(T-s)^{\beta-1} \eta(s, x(s)) d s \mid \\
\leq & \frac{\varepsilon}{\Gamma(\beta)} \int_{0}^{t}(t-s)^{\beta-1} \phi(s) d s \\
\leq & \frac{\varepsilon}{\Gamma(\beta)}\left(\int_{0}^{t}(t-s)^{\frac{\beta-1}{1-\gamma}} d s\right)^{1-\gamma}\left(\int_{0}^{t}(\phi(s))^{\frac{1}{\gamma}} d s\right)^{\gamma} \\
\leq & \frac{\varepsilon}{\Gamma(\beta)}\left(\frac{1-\gamma}{\beta-\gamma}\right)^{1-\gamma} t^{\beta-\gamma} c_{\phi} \phi(t)
\end{aligned}
$$




$$
\begin{aligned}
& \leq\left[\frac{c_{\phi}}{\Gamma(\beta)}\left(\frac{1-\gamma}{\beta-\gamma}\right)^{1-\gamma} s_{0}^{\beta-\gamma}\right] \varepsilon \phi(t) \\
& \leq\left[\frac{c_{\phi}}{\Gamma(\beta)}\left(\frac{1-\gamma}{\beta-\gamma}\right)^{1-\gamma} s_{0}^{\beta-\gamma}\right] \varepsilon(\phi(t)+\psi) ;
\end{aligned}
$$

for $t \in\left(s_{k-1}, t_{k}\right], k=1,2, \ldots, m$, we have

$$
\left|x(t)-I_{s_{k-1}, t_{k}}^{\beta} \xi_{k}(t, x(t))\right| \leq \varepsilon \psi
$$

for $t \in\left(t_{k}, s_{k}\right], k=1,2, \ldots, m$, we have

$$
\begin{aligned}
\mid x(t) & -\frac{1}{\Gamma(\beta)} \int_{0}^{t}(t-s)^{\beta-1} f\left(s, x(s),{ }^{c} D_{0, t}^{\beta} x(s)\right) d s-\frac{1}{\Gamma(\beta)} \int_{s_{k-1}}^{t_{k}}\left(t_{k}-s\right)^{\beta-1} \xi_{k}(s, x(s)) d s \\
& +\frac{1}{\Gamma(\beta)} \int_{0}^{t_{k}}\left(t_{k}-s\right)^{\beta-1} f\left(s, x(s),{ }^{c} D_{0, t}^{\beta} x(s)\right) d s \mid \\
\leq & \frac{\varepsilon}{\Gamma(\beta)} \int_{0}^{t}(t-s)^{\beta-1} \phi(s) d s+\frac{\varepsilon}{\Gamma(\beta)} \int_{0}^{t_{k}}\left(t_{k}-s\right)^{\beta-1} \phi(s) d s+\frac{\varepsilon \psi\left(t_{k}-s_{k-1}\right)^{\beta}}{\Gamma(\beta+1)} \\
\leq & \frac{\varepsilon}{\Gamma(\beta)}\left(\int_{0}^{t}(t-s)^{\frac{\beta-1}{1-\gamma}} d s\right)^{1-\gamma}\left(\int_{0}^{t}(\phi(s))^{\frac{1}{\gamma}} d s\right)^{\gamma} \\
& +\frac{\varepsilon}{\Gamma(\beta)}\left(\int_{0}^{t_{k}}\left(t_{k}-s\right)^{\frac{\beta-1}{1-\gamma}} d s\right)^{1-\gamma}\left(\int_{0}^{t_{k}}(\phi(s))^{\frac{1}{\gamma}} d s\right)^{\gamma}+\frac{\varepsilon \psi\left(t_{k}-s_{k-1}\right)^{\beta}}{\Gamma(\beta+1)} \\
\leq & \frac{\varepsilon}{\Gamma(\beta)}\left(\frac{1-\gamma}{\beta-\gamma}\right)^{1-\gamma} t^{\beta-\gamma} c_{\phi} \phi(t)+\frac{\varepsilon}{\Gamma(\beta)}\left(\frac{1-\gamma}{\beta-\gamma}\right)^{1-\gamma} t_{k}^{\beta-\gamma} c_{\phi} \phi(t)+\frac{\varepsilon \psi\left(t_{k}-s_{k-1}\right)^{\beta}}{\Gamma(\beta+1)} \\
\leq & \frac{\varepsilon}{\Gamma(\beta)}\left(\frac{1-\gamma}{\beta-\gamma}\right)^{1-\gamma} T^{\beta-\gamma} c_{\phi} \phi(t)+\frac{\varepsilon}{\Gamma(\beta)}\left(\frac{1-\gamma}{\beta-\gamma}\right)^{1-\gamma} t_{k}^{\beta-\gamma} c_{\phi} \phi(t)+\frac{\varepsilon \psi\left(t_{k}-s_{k-1}\right)^{\beta}}{\Gamma(\beta+1)} \\
\leq & \frac{c_{\phi}}{\Gamma(\beta)}\left(\frac{1-\gamma}{\beta-\gamma}\right)^{\beta-\gamma}\left(T^{\beta-\gamma}+t_{k}^{\beta-\gamma}\right) \varepsilon \phi(t)+\frac{\varepsilon \psi\left(t_{k}-s_{k-1}\right)^{\beta}}{\Gamma(\beta+1)} \\
\leq & \left.\frac{c_{\phi}}{\Gamma(\beta)}\left(\frac{1-\gamma}{\beta-\gamma}\right)^{\beta-\gamma}\left(T^{\beta-\gamma}+t_{k}^{\beta-\gamma}\right)+\frac{\left(t_{k}-s_{k-1}\right)^{\beta}}{\Gamma(\beta+1)}\right]^{\varepsilon(\phi(t)+\psi) .}
\end{aligned}
$$

Using the definition of $\Lambda$ given in Eq. (4.5), from the above cases we get

$$
|x(t)-(\Lambda x)(t)| \leq\left[\frac{c_{\phi}}{\Gamma(\beta)}\left(\frac{1-\gamma}{\beta-\gamma}\right)^{\beta-\gamma}\left(T^{\beta-\gamma}+t_{k}^{\beta-\gamma}\right)+\frac{\left(t_{k}-s_{k-1}\right)^{\beta}}{\Gamma(\beta+1)}\right] \varepsilon(\phi(t)+\psi)
$$

that is,

$$
d(x, \Lambda x) \leq \frac{c_{\phi}}{\Gamma(\beta)}\left(\frac{1-\gamma}{\beta-\gamma}\right)^{\beta-\gamma}\left(T^{\beta-\gamma}+t_{k}^{\beta-\gamma}\right)+\frac{\left(t_{k}-s_{k-1}\right)^{\beta}}{\Gamma(\beta+1)}, \quad t \in J
$$

and $t_{k}, s_{k-1}$ are considered to be maximum values. Hence, in view of Theorem 2.16(c) together with (4.7), we obtain

$$
d\left(x, x_{0}\right) \leq \frac{d(x, \Lambda x)}{1-L} \leq \frac{\left[\frac{c_{\phi}}{\Gamma(\beta)}\left(\frac{1-\gamma}{\beta-\gamma}\right)^{\beta-\gamma}\left(T^{\beta-\gamma}+t_{k}^{\beta-\gamma}\right)+\frac{\left(t_{k}-s_{k-1}\right)^{\beta}}{\Gamma(\beta+1)}\right]}{1-L}
$$


implies that

$$
\left|x(t)-x_{0}(t)\right| \leq \frac{\left[\frac{c_{\phi}}{\Gamma(\beta)}\left(\frac{1-\gamma}{\beta-\gamma}\right)^{\beta-\gamma}\left(T^{\beta-\gamma}+t_{k}^{\beta-\gamma}\right)+\frac{\left(t_{k}-s_{k-1}\right)^{\beta}}{\Gamma(\beta+1)}\right] \varepsilon(\phi(t)+\psi)}{1-L}
$$

for all $t \in J, 0<\gamma<\beta \leq 1$, where $t_{k}, s_{k-1}$ are considered to be maximum values and $L<1$.

Hence problem (1.3) is Ulam-Hyers-Rassias stable with respect to $(\phi, \psi)$.

Remark 4.2 By choosing $\varepsilon=1$, problem (1.3) is generalized Ulam-Hyers-Rassias stable with respect to $(\phi, \psi)$, in view of Definition 2.9.

Theorem 4.3 Let $c_{f, \beta, g}>0$ be a real constant. Assume that $\left(A_{1}\right)-\left(A_{4}\right)$ are true and a function $x \in \hat{\mathfrak{B}}$ satisfies inequality (2.1), then there exists a unique continuous function $x_{0}: J \rightarrow \mathbb{R}$ satisfying Eq. (4.1) and

$$
\left|x(t)-x_{0}(t)\right| \leq c_{f, \beta, g} \varepsilon, \quad t \in J,
$$

with

$$
c_{f, \beta, g}=\frac{\left[\frac{2}{\Gamma(\beta+1)}\left\{T^{\beta}+\left(t_{k}-s_{k-1}\right)^{\beta}+t_{k}^{\beta}\right\}\right]}{1-L},
$$

where $t_{k}, s_{k-1}$ are considered to be maximum values and

$$
L=\max \left\{\frac{\bar{K}_{f}}{\Gamma(\beta+1)}\left(s_{k}^{\beta}+t_{k}^{\beta}\right)+\frac{L_{\xi_{k}}}{\Gamma(\beta+1)}\left(t_{k}-s_{k-1}\right)^{\beta} \mid k=1,2, \ldots, m\right\}<1,
$$

that is, problem (1.3) is Ulam-Hyers stable.

Proof First, define a set $Y$ of all piecewise continuous functions $p: J \rightarrow \mathbb{R}$ in the following way:

$$
Y=\{p: J \rightarrow \mathbb{R} \mid p \in \hat{\mathfrak{B}}\}
$$

and let us introduce a generalized complete metric on $Y$ as

$$
d(p, q)=\inf \left\{C_{1}+C_{2} \in[0,+\infty]|| p(t)-q(t) \mid \leq\left(C_{1}+C_{2}\right)(2 \varepsilon)\right\},
$$

for all $t \in J$, where

$$
C_{1} \in\{C \in[0, \infty]|| p(t)-q(t) \mid \leq C \varepsilon\}, \quad t \in\left(t_{k}, s_{k}\right], k=0,1, \ldots, m,
$$

and

$$
C_{2} \in\{C \in[0, \infty]|| p(t)-q(t) \mid \leq C \varepsilon\}, \quad t \in\left(s_{k-1}, t_{k}\right], k=1, \ldots, m .
$$


Define an operator $\Lambda: J \rightarrow \mathbb{R}$ by

$$
(\Lambda y)(t)= \begin{cases}\frac{1}{\Gamma(\beta)} \int_{0}^{t}(t-s)^{\beta-1} f\left(s, y(s),{ }^{c} D_{0, t}^{\beta} y(s)\right) d s & \\ \quad+\frac{1}{\Gamma(\beta)} \int_{0}^{T}(T-s)^{\beta-1} \eta(s, y(s)) d s, & t \in\left[0, s_{0}\right], \\ I_{s_{k-1}, t_{k}}^{\beta}\left(\xi_{k}(t, y(t))\right), & k=1,2, \ldots, m, \\ \frac{1}{\Gamma(\beta)} \int_{0}^{t}(t-s)^{\beta-1} f\left(s, y(s),{ }^{c} D_{0, t}^{\beta} y(s)\right) d s & \\ \quad+\frac{1}{\Gamma(\beta)} \int_{s_{k-1}}^{t_{k}}\left(t_{k}-s\right)^{\beta-1} \xi_{k}(s, y(s)) d s & \\ -\frac{1}{\Gamma(\beta)} \int_{0}^{t_{k}}\left(t_{k}-s\right)^{\beta-1} f\left(s, y(s),{ }^{c} D_{0, t}^{\beta} y(s)\right) d s, & t \in\left(t_{k}, s_{k}\right],\end{cases}
$$

for all $y \in Y$. Clearly, $\Lambda$ is a well-defined operator, because $y$ and $f$ are continuous functions.

Next, we have to prove that $\Lambda$ is strictly contractive on $Y$. For any $p, q \in Y$ and $C_{1}, C_{2} \in$ $[0,+\infty]$, using Eq. (4.10), we have

$$
|p(t)-q(t)| \leq \begin{cases}C_{1} \varepsilon, & t \in\left(t_{k}, s_{k}\right], k=0,1, \ldots, m \\ C_{2} \varepsilon, & t \in\left(s_{k-1}, t_{k}\right], k=1,2, \ldots, m\end{cases}
$$

It follows from the property that the Caputo fractional derivative of a constant is always zero, Eqs. (4.11), (4.12) and from assumptions $\left(A_{2}\right)-\left(A_{4}\right)$ that:

Case 1: For $t \in\left[0, s_{0}\right]$, we have

$$
\begin{aligned}
|(\Lambda p)(t)-(\Lambda q)(t)|= & \frac{1}{\Gamma(\beta)} \int_{0}^{t}(t-s)^{\beta-1} f\left(s, p(s),{ }^{c} D_{0, t}^{\beta} p(s)\right) d s \\
& +\frac{1}{\Gamma(\beta)} \int_{0}^{T}(T-s)^{\beta-1} \eta(s, p(s)) d s \\
& -\frac{1}{\Gamma(\beta)} \int_{0}^{t}(t-s)^{\beta-1} f\left(s, q(s),{ }^{c} D_{0, t}^{\beta} q(s)\right) d s \\
& -\frac{1}{\Gamma(\beta)} \int_{0}^{T}(T-s)^{\beta-1} \eta(s, q(s)) d s \mid \\
\leq & \frac{1}{\Gamma(\beta)} \int_{0}^{t}(t-s)^{\beta-1}\left|f\left(s, p(s),{ }^{c} D_{0, t}^{\beta} p(s)\right)-f\left(s, q(s),{ }^{c} D_{0, t}^{\beta} q(s)\right)\right| d s \\
& +\frac{1}{\Gamma(\beta)} \int_{0}^{T}(T-s)^{\beta-1}|\eta(s, p(s))-\eta(s, q(s))| d s \\
\leq & \frac{1}{\Gamma(\beta)} \int_{0}^{t}(t-s)^{\beta-1}\left[\overline{K_{f}}|p(s)-q(s)|+\overline{L_{k}}\left|{ }^{c} D_{0, t}^{\beta} p(s)-{ }^{c} D_{0, t}^{\beta} q(s)\right|\right] d s \\
& +\frac{1}{\Gamma(\beta)} \int_{0}^{T}(T-s)^{\beta-1}\left[L_{\eta}|p(s)-q(s)|\right] d s \\
\leq & \frac{\overline{K_{f}}}{\Gamma(\beta)} \int_{0}^{t}(t-s)^{\beta-1} C_{1} \varepsilon d s+\frac{L_{f}}{\Gamma(\beta)} \int_{0}^{t}(t-s)^{\beta-1}\left({ }^{c} D_{0, t}^{\beta} C_{1} \varepsilon\right) d s \\
& +\frac{L_{\eta}}{\Gamma(\beta)} \int_{0}^{T}(T-s)^{\beta-1} C_{1} \varepsilon d s, \\
& \\
& \\
&
\end{aligned}
$$


Zada et al. Advances in Difference Equations ( 2017) 2017:317

Page 19 of 26

which implies that

$$
\begin{aligned}
|(\Lambda p)(t)-(\Lambda q)(t)| & \leq \frac{\bar{K}_{f} C_{1} \varepsilon}{\Gamma(\beta+1)} t^{\beta}+\frac{L_{\eta} C_{1} \varepsilon}{\Gamma(\beta+1)} T^{\beta} \\
& \leq\left(\frac{\bar{K}_{f} s_{0}^{\beta}+L_{\eta} T^{\beta}}{\Gamma(\beta+1)}\right) C_{1} \varepsilon .
\end{aligned}
$$

Case 2: For $t \in\left(s_{k-1}, t_{k}\right], k=1,2, \ldots, m$, we have

$$
\begin{aligned}
|(\Lambda p)(t)-(\Lambda q)(t)| & =\left|I_{s_{k-1}, t_{k}}^{\beta} \xi_{k}(t, p(t))-I_{s_{k-1}, t_{k}}^{\beta} \xi_{k}(t, q(t))\right| \\
& \leq L_{\xi_{k}}|p(t)-q(t)| \leq L_{\xi_{k}} C_{2} \varepsilon .
\end{aligned}
$$

Case 3(a): For $t \in\left(t_{k}, s_{k}\right]$ and $s \in\left(t_{k}, s_{k}\right], k=1,2, \ldots, m$, we have

$$
\begin{aligned}
& |(\Lambda p)(t)-(\Lambda q)(t)|=\mid \frac{1}{\Gamma(\beta)} \int_{0}^{t}(t-s)^{\beta-1} f\left(s, p(s),{ }^{c} D_{0, t}^{\beta} p(s)\right) d s \\
& +\frac{1}{\Gamma(\beta)} \int_{s_{k-1}}^{t_{k}}\left(t_{k}-s\right)^{\beta-1} \xi_{k}(s, p(s)) d s \\
& -\frac{1}{\Gamma(\beta)} \int_{0}^{t_{k}}\left(t_{k}-s\right)^{\beta-1} f\left(s, p(s),{ }^{c} D_{0, t}^{\beta} p(s)\right) d s \\
& -\frac{1}{\Gamma(\beta)} \int_{0}^{t}(t-s)^{\beta-1} f\left(s, q(s),{ }^{c} D_{0, t}^{\beta} q(s)\right) d s \\
& -\frac{1}{\Gamma(\beta)} \int_{s_{k-1}}^{t_{k}}\left(t_{k}-s\right)^{\beta-1} \xi_{k}(s, q(s)) d s \\
& +\frac{1}{\Gamma(\beta)} \int_{0}^{t_{k}}\left(t_{k}-s\right)^{\beta-1} f\left(s, q(s),{ }^{c} D_{0, t}^{\beta} q(s)\right) d s \mid \\
& \leq \mid \frac{1}{\Gamma(\beta)} \int_{0}^{t}(t-s)^{\beta-1} f\left(s, p(s),{ }^{c} D_{0, t}^{\beta} p(s)\right) d s \\
& -\frac{1}{\Gamma(\beta)} \int_{0}^{t}(t-s)^{\beta-1} f\left(s, q(s),{ }^{c} D_{0, t}^{\beta} q(s)\right) d s \\
& +\mid \frac{1}{\Gamma(\beta)} \int_{s_{k-1}}^{t_{k}}\left(t_{k}-s\right)^{\beta-1} \xi_{k}(s, p(s)) d s \\
& -\frac{1}{\Gamma(\beta)} \int_{s_{k-1}}^{t_{k}}\left(t_{k}-s\right)^{\beta-1} \xi_{k}(s, q(s)) d s \mid \\
& +\mid \frac{1}{\Gamma(\beta)} \int_{0}^{t_{k}}\left(t_{k}-s\right)^{\beta-1} f\left(s, q(s),{ }^{c} D_{0, t}^{\beta} q(s)\right) d s \\
& -\frac{1}{\Gamma(\beta)} \int_{0}^{t_{k}}\left(t_{k}-s\right)^{\beta-1} f\left(s, p(s),{ }^{c} D_{0, t}^{\beta} p(s)\right) d s \mid \\
& \leq \frac{\bar{K}_{f}}{\Gamma(\beta+1)}\left(t^{\beta}+t_{k}^{\beta}\right) C_{1} \varepsilon+\frac{L_{\xi_{k}}}{\Gamma(\beta+1)}\left(t_{k}-s_{k-1}\right)^{\beta} C_{2} \varepsilon \\
& \leq\left[\frac{\bar{K}_{f}}{\Gamma(\beta+1)}\left(s_{k}^{\beta}+t_{k}^{\beta}\right)+\frac{L_{\xi_{k}}}{\Gamma(\beta+1)}\left(t_{k}-s_{k-1}\right)^{\beta}\right]\left(C_{1}+C_{2}\right)(2 \varepsilon) \text {. }
\end{aligned}
$$


Case 3(b): For $t \in\left(t_{k}, s_{k}\right]$ and $s \in\left(s_{k-1}, t_{k}\right], k=1,2, \ldots, m$, we have

$$
\begin{aligned}
& |(\Lambda p)(t)-(\Lambda q)(t)|=\mid \frac{1}{\Gamma(\beta)} \int_{0}^{t}(t-s)^{\beta-1} f\left(s, p(s),{ }^{c} D_{0, t}^{\beta} p(s)\right) d s \\
& +\frac{1}{\Gamma(\beta)} \int_{s_{k-1}}^{t_{k}}\left(t_{k}-s\right)^{\beta-1} \xi_{k}(s, p(s)) d s \\
& -\frac{1}{\Gamma(\beta)} \int_{0}^{t_{k}}\left(t_{k}-s\right)^{\beta-1} f\left(s, p(s),{ }^{c} D_{0, t}^{\beta} p(s)\right) d s \\
& -\frac{1}{\Gamma(\beta)} \int_{0}^{t}(t-s)^{\beta-1} f\left(s, q(s),{ }^{c} D_{0, t}^{\beta} q(s)\right) d s \\
& -\frac{1}{\Gamma(\beta)} \int_{s_{k-1}}^{t_{k}}\left(t_{k}-s\right)^{\beta-1} \xi_{k}(s, q(s)) d s \\
& +\frac{1}{\Gamma(\beta)} \int_{0}^{t_{k}}\left(t_{k}-s\right)^{\beta-1} f\left(s, q(s),{ }^{c} D_{0, t}^{\beta} q(s)\right) d s \\
& \leq \mid \frac{1}{\Gamma(\beta)} \int_{0}^{t}(t-s)^{\beta-1} f\left(s, p(s),{ }^{c} D_{0, t}^{\beta} p(s)\right) d s \\
& -\frac{1}{\Gamma(\beta)} \int_{0}^{t}(t-s)^{\beta-1} f\left(s, q(s),{ }^{c} D_{0, t}^{\beta} q(s)\right) d s \mid \\
& +\mid \frac{1}{\Gamma(\beta)} \int_{s_{k-1}}^{t_{k}}\left(t_{k}-s\right)^{\beta-1} \xi_{k}(s, p(s)) d s \\
& -\frac{1}{\Gamma(\beta)} \int_{s_{k-1}}^{t_{k}}\left(t_{k}-s\right)^{\beta-1} \xi_{k}(s, q(s)) d s \\
& +\mid \frac{1}{\Gamma(\beta)} \int_{0}^{t_{k}}\left(t_{k}-s\right)^{\beta-1} f\left(s, q(s),{ }^{c} D_{0, t}^{\beta} q(s)\right) d s \\
& -\frac{1}{\Gamma(\beta)} \int_{0}^{t_{k}}\left(t_{k}-s\right)^{\beta-1} f\left(s, p(s),{ }^{c} D_{0, t}^{\beta} p(s)\right) d s \mid \\
& \leq \frac{\bar{K}_{f}}{\Gamma(\beta+1)}\left(t^{\beta}+t_{k}^{\beta}\right) C_{2} \varepsilon+\frac{L_{\xi_{k}}}{\Gamma(\beta+1)}\left(t_{k}-s_{k-1}\right)^{\beta} C_{2} \varepsilon \\
& \leq\left[\frac{\bar{K}_{f}}{\Gamma(\beta+1)}\left(s_{k}^{\beta}+t_{k}^{\beta}\right)+\frac{L_{\xi_{k}}}{\Gamma(\beta+1)}\left(t_{k}-s_{k-1}\right)^{\beta}\right]\left(C_{1}+C_{2}\right)(2 \varepsilon) \text {. }
\end{aligned}
$$

From above, we obtain

$$
|(\Lambda p)(t)-(\Lambda q)(t)| \leq L\left(C_{1}+C_{2}\right)(2 \varepsilon), \quad t \in J
$$

that is,

$$
d(\Lambda p, \Lambda q) \leq L\left(C_{1}+C_{2}\right)(2 \varepsilon), \quad t \in J
$$

which implies that $\Lambda$ is strictly contractive on $Y$ for any $p, q \in Y$ and $L<1$. 
Let $p_{0} \in Y$, it follows from the continuity property of $p_{0}$ and $\Lambda p_{0}$ that there exists a constant $0<\vartheta_{1}<\infty$ such that

$$
\begin{aligned}
\left|\left(\Lambda p_{0}\right)(t)-p_{0}(t)\right|= & \mid \frac{1}{\Gamma(\beta)} \int_{0}^{t}(t-s)^{\beta-1} f\left(s, p_{0}(s),{ }^{c} D_{0, t}^{\beta} p_{0}(s)\right) d s \\
& +\frac{1}{\Gamma(\beta)} \int_{0}^{T}(T-s)^{\beta-1} \eta\left(s, p_{0}(s)\right) d s-p_{0}(t) \mid \\
\leq & \vartheta_{1}(2 \varepsilon), \quad t \in\left[0, s_{0}\right],
\end{aligned}
$$

also there exists a constant $0<\vartheta_{2}<\infty$ such that

$$
\left|\left(\Lambda p_{0}\right)(t)-p_{0}(t)\right|=\left|I_{s_{k-1}, t_{k}}^{\beta} \xi_{k}\left(t, p_{0}(t)\right)-p_{0}(t)\right| \leq \vartheta_{2}(2 \varepsilon), \quad t \in\left(s_{k-1}, t_{k}\right], k=1,2, \ldots, m .
$$

Furthermore, we also can obtain a constant $\vartheta_{3}$ between 0 and $\infty$ such that

$$
\begin{aligned}
\left|\left(\Lambda p_{0}\right)(t)-p_{0}(t)\right|= & \mid \frac{1}{\Gamma(\beta)} \int_{0}^{t}(t-s)^{\beta-1} f\left(s, p_{0}(s),{ }^{c} D_{0, t}^{\beta} p_{0}(s)\right) d s \\
& +\frac{1}{\Gamma(\beta)} \int_{s_{k-1}}^{t_{k}}\left(t_{k}-s\right)^{\beta-1} \xi_{k}\left(s, p_{0}(s)\right) d s \\
& -\frac{1}{\Gamma(\beta)} \int_{0}^{t_{k}}\left(t_{k}-s\right)^{\beta-1} f\left(s, p_{0}(s),{ }^{c} D_{0, t}^{\beta} p_{0}(s)\right) d s-p_{0}(t) \mid \\
\leq & \vartheta_{3}(2 \varepsilon), \quad t \in\left(t_{k}, s_{k}\right], k=1,2, \ldots, m .
\end{aligned}
$$

As $f, \xi_{k}$ and $p_{0}$ are bounded on $J$ and $2 \varepsilon>0$, therefore Eq. (4.10) implies that

$$
d\left(\Lambda p_{0}, p_{0}\right)<\infty
$$

According to Theorem 2.16(a), there exists a continuous function $x_{0}: J \rightarrow \mathbb{R}$ such that $\Lambda^{n} p_{0} \rightarrow x_{0}$ in $(Y, d)$ as $n \rightarrow \infty$ and $\Lambda x_{0}=x_{0}$, that is, $x_{0}$ satisfies Eq. (4.1) for all $t \in J$.

Next, we check that

$$
\left\{p \in Y \mid d\left(p_{0}, p\right)<\infty\right\}=Y .
$$

For any $p \in Y$, since $p$ and $p_{0}$ are bounded on $J$ and $2 \varepsilon>0$, there exists a constant $0<\vartheta_{p}<$ $\infty$ such that

$$
\left|p_{0}(t)-p(t)\right| \leq \vartheta_{p}(2 \varepsilon), \quad t \in J
$$

Thus we obtain $d\left(\Lambda p_{0}, p_{0}\right)<\infty$ for all $p \in Y$, that is,

$$
\left\{p \in Y \mid d\left(p_{0}, p\right)<\infty\right\}=Y .
$$

Hence, in view of Theorem 2.16(b), we conclude that $x_{0}$ is the unique continuous function satisfying Eq. (4.1). 
On the other hand, it follows from the definition of $\Lambda$ and Remark 3.2 that

$$
|x(t)-(\Lambda x)(t)| \leq\left[\frac{2}{\Gamma(\beta+1)}\left\{T^{\beta}+\left(t_{k}-s_{k-1}\right)^{\beta}+t_{k}^{\beta}\right\}\right] \varepsilon,
$$

that is,

$$
d(x, \Lambda x) \leq \frac{2}{\Gamma(\beta+1)}\left\{T^{\beta}+\left(t_{k}-s_{k-1}\right)^{\beta}+t_{k}^{\beta}\right\}, \quad t \in J,
$$

and $t_{k}, s_{k-1}$ are considered to be maximum values. From above Eq. (4.13) and Theorem 2.16(c), we have

$$
d\left(x, x_{0}\right) \leq \frac{d(x, \Lambda x)}{1-L} \leq \frac{\frac{2}{\Gamma(\beta+1)}\left\{T^{\beta}+\left(t_{k}-s_{k-1}\right)^{\beta}+t_{k}^{\beta}\right\}}{1-L},
$$

which implies that

$$
\left|x(t)-x_{0}(t)\right| \leq c_{f, \beta, g} \varepsilon
$$

for all $t \in J$ and $0<\beta \leq 1$, with

$$
c_{f, \beta, g}=\frac{\left[\frac{2}{\Gamma(\beta+1)}\left\{T^{\beta}+\left(t_{k}-s_{k-1}\right)^{\beta}+t_{k}^{\beta}\right\}\right]}{1-L},
$$

where $t_{k}, s_{k-1}$ are considered to be maximum values and

$$
L=\max \left\{\frac{\bar{K}_{f}}{\Gamma(\beta+1)}\left(s_{k}^{\beta}+t_{k}^{\beta}\right)+\frac{L_{\xi_{k}}}{\Gamma(\beta+1)}\left(t_{k}-s_{k-1}\right)^{\beta} \mid k=1,2, \ldots, m\right\}<1 .
$$

Hence problem (1.3) is Ulam-Hyers stable.

Remark 4.4 If $\lambda_{f, \beta, g}(\varepsilon)=c_{f, \beta, g} \varepsilon$ with $\lambda_{f, \beta, g}(0)=0$, then problem (1.3) is generalized UlamHyers stable.

\section{Examples}

In this section we show the applicability of our main results by providing some examples.

Example 5.1 Consider

$$
\begin{cases}{ }^{c} D_{0, t}^{\frac{1}{2}} y(t)=\frac{|y(t)|+\left.\right|^{c} D_{0, t}^{\frac{1}{2}} y(t) \mid}{\left(1+e^{t}\right)\left(1+|y(t)|+\left|{ }^{c} D_{0, t}^{\frac{1}{2}} y(t)\right|\right)}, & t \in(0,1] \cup(2,3], \\ y(t)=I_{1,2}^{\frac{1}{2}} \xi_{1}(t, y(t))=\frac{1}{\Gamma\left(\frac{1}{2}\right)} \int_{1}^{2}(2-s)^{\frac{-1}{2}} \log |y(s)| d s, & t \in(1,2], \\ y(0)=I_{0,3}^{\frac{1}{2}} \eta(t, y(t))=\frac{1}{\Gamma\left(\frac{1}{2}\right)} \int_{0}^{3}(3-s)^{\frac{-1}{2}} \cos |y(s)| d s, & \end{cases}
$$

where ${ }^{c} D_{0, t}^{\frac{1}{2}}$ is the Caputo fractional derivative of order $\frac{1}{2}$ with lower limit $0,0=t_{0}<s_{0}=$ $1<t_{1}=2<s_{1}=3$ and $J=[0,3]$. 
And

$$
\begin{cases}\left|{ }^{c} D_{0, t^{\frac{1}{2}}}^{2} x(t)-\frac{|x(t)|+\left.\right|^{c} D_{0, t}^{\frac{1}{2}} x(t) \mid}{\left(1+e^{t}\right)\left(1+|x(t)|+\left.\right|^{c} D_{0, t}^{\frac{1}{2}} x(t) \mid\right)}\right| \leq \varepsilon \phi(t), & t \in(0,1] \cup(2,3], \\ \left|x(t)-\frac{1}{\Gamma\left(\frac{1}{2}\right)} \int_{1}^{2}(2-s)^{\frac{-1}{2}} \log \right| x(s)|d s| \leq \varepsilon \psi, & t \in(1,2],\end{cases}
$$

with condition

$$
x(0)=I_{0,3}^{\frac{1}{2}} \eta(t, x(t))=\frac{1}{\Gamma\left(\frac{1}{2}\right)} \int_{0}^{3}(3-s)^{\frac{-1}{2}} \cos |x(s)| d s .
$$

Denote the continuous functions $f, \xi_{1}$ and $\eta$ as follows:

$$
f\left(t, y(t),{ }^{c} D_{0, t}^{\beta} y(t)\right)=\frac{|y(t)|+\left|{ }^{c} D_{0, t}^{\frac{1}{2}} y(t)\right|}{\left(1+e^{t}\right)\left(1+|y(t)|+\left|{ }^{c} D_{0, t}^{\frac{1}{2}} y(t)\right|\right)}, \quad t \in(0,1] \cup(2,3]
$$

with $\bar{K}_{f}=\frac{1}{9}$ and $\bar{L}_{f}=\frac{1}{3}$,

$$
\xi_{1}(t, y(t))=\log |y(t)|, \quad t \in(1,2]
$$

with $L_{\xi_{k}}=0.1$, for $k=0,1$ and

$$
\eta(t, y(t))=\cos |y(t)|, \quad t \in[0,3]
$$

with $L_{\eta}=0.2$.

We put $\gamma=\frac{1}{5}, \varepsilon=\psi=\frac{1}{2}, \phi(t)=8 e^{t}$ and $c_{\phi}=c_{\beta}=\frac{1}{8}$, we have

$$
\left(\int_{0}^{t}\left(e^{s}\right)^{3} d s\right)^{\frac{1}{3}} \leq \frac{1}{8}\left(8 e^{t}\right)=e^{t}, \quad t \in[0,3]
$$

and

$$
\left(\int_{0}^{t}\left({ }^{c} D_{0, t}^{\frac{1}{2}} e^{s}\right)^{3} d s\right)^{\frac{1}{3}} \leq \frac{1}{8}\left(8 e^{t}\right)=e^{t}, \quad t \in[0,3] .
$$

From the above considerations, we clearly see that $\left(A_{1}\right)-\left(A_{5}\right)$ are satisfied.

As

$$
\begin{aligned}
& L_{1}=\max \left\{\frac{\bar{K}_{f} c_{\phi}+\bar{L}_{f} c_{\beta}}{\Gamma(\beta)}\left(\frac{1-\gamma}{\beta-\gamma}\right)^{1-\gamma}\left(s_{k}^{\beta-\gamma}+t_{k}^{\beta-\gamma}\right)+L_{\xi_{k}} \mid k=0,1\right\}, \\
& L_{2}=\left\{\frac{\bar{K}_{f}}{\Gamma(\beta+1)}\left(s_{1}^{\beta}+t_{1}^{\beta}\right)+L_{\xi_{1}}\right\} .
\end{aligned}
$$

By putting the above values, we get $L_{1}=\max \{0.1688214,0.2539802\} \approx 0.25398$ and $L_{2}=$ $0.4886639641 \approx 0.5$, so $L=0.5<1$. 
By Theorem 4.1, there exists a unique solution $x_{0}:[0,3] \rightarrow \mathbb{R}$ such that

$$
x_{0}(t)=\left\{\begin{array}{cc}
\frac{1}{\Gamma\left(\frac{1}{2}\right)} \int_{0}^{t}(t-s)^{\frac{-1}{2}}\left(\frac{\left|x_{0}(s)\right|+\left.\right|^{c} D_{0, t^{\frac{1}{2}}}^{x_{0}(s) \mid}}{\left(1+e^{s}\right)\left(1+\left|x_{0}(s)\right|+\left.\right|^{c} D_{0, t^{\frac{1}{2}}}^{\left.x_{0}(s) \mid\right)}\right) d s}\right. & \\
\quad+\frac{1}{\Gamma\left(\frac{1}{2}\right)} \int_{0}^{3}(3-s)^{\frac{-1}{2}} \cos \left|x_{0}(s)\right| d s, & t \in[0,1] \\
I_{1,2}^{\frac{1}{2}}\left(\xi_{1}\left(t, x_{0}(t)\right)\right)=\frac{1}{\Gamma\left(\frac{1}{2}\right)} \int_{1}^{2}(2-s)^{\frac{-1}{2}} \log \left|x_{0}(s)\right| d s, & t \in(1,2] \\
\frac{1}{\Gamma\left(\frac{1}{2}\right)} \int_{0}^{t}(t-s)^{\frac{-1}{2}}\left(\frac{\left|x_{0}(s)\right|+{ }^{c} D_{0, t}^{\frac{1}{2}} x_{0}(s) \mid}{\left(1+e^{s}\right)\left(1+\left|x_{0}(s)\right|+{ }^{c} D_{0, t}^{\frac{1}{2}} x_{0}(s) \mid\right)}\right) d s & \\
\quad+\frac{1}{\Gamma\left(\frac{1}{2}\right)} \int_{0}^{3}(3-s)^{\frac{-1}{2}} \cos \left|x_{0}(s)\right| d s & \\
+\frac{1}{\Gamma\left(\frac{1}{2}\right)} \int_{0}^{2}(2-s)^{\frac{-1}{2}}\left(\frac{\left|x_{0}(s)\right|+\left.\right|^{c} D_{0, t}^{\frac{1}{2}} x_{0}(s) \mid}{\left(1+e^{s}\right)\left(1+\left|x_{0}(s)\right|+\left|{ }^{c} D_{0, t}^{\frac{1}{2}} x_{0}(s)\right|\right)}\right) d s, & t \in(2,3]
\end{array}\right.
$$

and

$$
\left|x(t)-x_{0}(t)\right| \leq \frac{[1.38] \frac{1}{2}\left(8 e^{t}+\frac{1}{2}\right)}{1-0.5} \approx 2.76\left\{\frac{1}{2}\left(8 e^{t}+\frac{1}{2}\right)\right\}
$$

which implies that problem (5.1) is Ulam-Hyers-Rassias stable.

Remark 5.2 If we set $\varepsilon=1$, then problem (5.1) is generalized Ulam-Hyers-Rassias stable.

Example 5.3 Consider problem (5.1) and

$$
\begin{cases}\left|{ }^{c} D_{0, t}^{\frac{1}{2}} x(t)-f\left(t, x(t),{ }^{c} D_{0, t}^{\frac{1}{2}} x(t)\right)\right| \leq \varepsilon, & t \in(0,1] \cup(2,3] \\ \left|x(t)-I_{1,2}^{\frac{1}{2}} \xi_{1}(t, x(t))\right| \leq \varepsilon, & t \in(1,2]\end{cases}
$$

with condition

$$
x(0)=I_{0,3}^{\frac{1}{2}} \eta(t, x(t))=\frac{1}{\Gamma\left(\frac{1}{2}\right)} \int_{0}^{3}(3-s)^{\frac{-1}{2}} \cos |x(s)| d s .
$$

The continuous functions $f, \xi_{1}$ and $\eta$ are defined as follows:

$$
f\left(t, y(t),{ }^{c} D_{0, t}^{\frac{1}{2}} x(t)\right)=\frac{|x(t)|+\left|{ }^{c} D_{0, t}^{\frac{1}{2}} x(t)\right|}{\left(1+e^{t}\right)\left(1+|x(t)|+\left|{ }^{c} D_{0, t}^{\frac{1}{2}} x(t)\right|\right)}, \quad t \in(0,1] \cup(2,3]
$$

with $\bar{K}_{f}=\frac{1}{9}$ and $\bar{L}_{f}=\frac{1}{3}$,

$$
\xi_{1}(t, x(t))=\log |x(t)|, \quad t \in(1,2]
$$

with $L_{\xi_{k}}=0.1$, for $k=0,1$ and

$$
\eta(t, y(t))=\cos |y(t)|, \quad t \in[0,3]
$$

with $L_{\eta}=0.2$. 
We put $\gamma=\frac{1}{5}$ and $\varepsilon$ is any positive real constant, i.e., $\varepsilon>0$. From the above considerations, we clearly see that $\left(A_{1}\right)-\left(A_{4}\right)$ are satisfied.

As we have

$$
L=\max \left\{\frac{\bar{K}_{f}}{\Gamma(\beta+1)}\left(s_{1}^{\beta}+t_{1}^{\beta}\right)+\frac{L_{\xi_{1}}}{\Gamma(\beta+1)}\left(t_{1}-s_{0}\right)^{\beta}\right\} .
$$

By putting the above values, we obtain $L \approx 0.506075<1$.

In view of Theorem 4.3 , there exists a unique solution $x_{0}:[0,3] \rightarrow \mathbb{R}$ satisfying Eq. (5.2) and

$$
\left|x(t)-x_{0}(t)\right| \leq c_{f, \beta, g} \varepsilon, \quad t \in J
$$

with

$$
c_{f, \beta, g}=\frac{\frac{2}{\Gamma\left(\frac{3}{2}\right)}\left(3^{\frac{1}{2}}+(2-1)^{\frac{1}{2}}+2^{\frac{1}{2}}\right)}{1-0.506075} \approx 18.95>0,
$$

which implies that

$$
\left|x(t)-x_{0}(t)\right| \leq(18.95) \varepsilon
$$

Thus problem (5.1) is Ulam-Hyers stable.

Remark 5.4 If we consider $\lambda_{f, \beta, g}(\varepsilon)=c_{f, \beta, g} \varepsilon$, i.e., $\lambda_{f, \beta, g}(\varepsilon)=(18.95) \varepsilon$ with $\lambda_{f, \beta, g}(0)=0$, then problem (5.1) is generalized Ulam-Hyers stable.

\section{Conclusion}

In view of generalized Diaz-Margolis's fixed point theorem, we established sufficient conditions for the existence and uniqueness of the solutions of problem (1.3) and proved different types of Ulam stability results for nonlinear implicit fractional differential equations of problem (1.3), taking into account the Caputo fractional derivative and its two properties, i.e., Caputo fractional derivative is a linear operator and its derivative of a constant is zero.

Acknowledgements

This work was supported by the National Natural Science Foundation of China (11571378).

Competing interests

The authors declare that they have no competing interests regarding this research work.

Authors' contributions

All the authors contributed equally and significantly in writing this paper. All the authors read and approved the final manuscript.

\section{Author details}

'Department of Mathematics, University of Peshawar, Peshawar, 25000, Pakistan. ${ }^{2}$ Department of Mathematics, Sun Yat-sen University, Guangzhou, 510275, P.R. China.

\section{Publisher's Note}

Springer Nature remains neutral with regard to jurisdictional claims in published maps and institutional affiliations.

Received: 19 June 2017 Accepted: 21 September 2017 Published online: 10 October 2017 


\section{References}

1. Leibnitz, GW: Leibnitzen's Mathematische Schriften, vol. 2, pp. 301-302. Georg Olm, Hildesheim (1962)

2. Lacroix, SF: Traite du Calcul Differentiel et du Calcul Integral, vol. 2, pp. 409-410. Mme. recourcier, Paris (1819)

3. Anastassiou, GA: Advances on Fractional Inequalities. Springer, New York (2011)

4. Abbas, S, Benchohra, M, N'Guerekata, GM: Topics in Fractional Differential Equations. Springer, New York (2012)

5. Abbas, S, Benchohra, M, N'Guerekata, GM: Advanced Fractional Differential and Integral Equations. Nova Publ. (Nova Science Publishers), New York (2014)

6. Podlubny, I: Fractional Differential Equations. Academic Press, San Diego (1999)

7. Samko, SG, Kilbas, AA, Marichev, Ol: Fractional Integrals and Derivatives, Theory and Applications. Gordon \& Breach, Yverdon (1993)

8. Benchohra, M, Lazreg, JE: Nonlinear fractional implicit differential equations. Commun. Appl. Anal. 17, 471-482 (2013)

9. Benchohra, M, Lazreg, JE: Existence and uniqueness results for nonlinear implicit fractional differential equations with boundary conditions. Rom. J. Math. Comput. Sci. 4, 60-72 (2014)

10. Hilfer, R: Applications of Fractional Calculus in Physics. World Scientific, Singapore (2000)

11. Lakshmikantham, V, Leela, S, Vasundhara, J: Theory of Fractional Dynamic Systems. Cambridge Academic Publishers, Cambridge (2009)

12. Ortigueira, MD: Fractional Calculus for Scientists and Engineers. Lecture Notes in Electrical Engineering. Springer, Dordrecht (2011)

13. Balachandran, K, Kiruthika, S: Existence of solutions of abstract fractional impulsive semilinear evolution equations. Electron. J. Qual. Theory Differ. Equ. 2010, Article ID 4 (2010)

14. Benchohra, M, Seba, D: Impulsive fractional differential equations in Banach spaces. Electron. J. Qual. Theory Differ. Equ. 2009, Article ID 8 (2009)

15. Kosmatov, N: Initial value problems of fractional order with fractional impulsive conditions. Results Math. 63, 1289-1310 (2013)

16. Wang, G, Zhang, L, Song, G: Systems of first order impulsive fractional differential equations with deviating arguments and nonlinear boundary conditions. Nonlinear Anal. TMA 74, 974-982 (2011)

17. Mardanov, MJ, Mahmudov, NI, Sharifov, YA: Existence and uniqueness theorems for impulsive fractional differential equations with two-point and integral boundary conditions. Sci. World J. 2014, Article ID 918730 (2014)

18. Ulam, SM: A Collection of Mathematical Problems. Interscience, New York (1968)

19. Hyers, DH: On the stability of the linear functional equation. Proc. Natl. Acad. Sci. USA 27(4), $222-224$ (1941)

20. Hyers, DH, Isac, G, Rassias, Th: Stability of Functional Equations in Several Variables. Birkhäuser, Boston (1998)

21. Li, T, Zada, A: Connections between Hyers-Ulam stability and uniform exponential stability of discrete evolution families of bounded linear operators over Banach spaces. Adv. Differ. Equ. 2016, Article ID 153 (2016)

22. Li, T, Zada, A, Faisal, S: Hyers-Ulam stability of $n$th order linear differential equations. J. Nonlinear Sci. Appl. 9, 2070-2075 (2016)

23. Rassias, ThM: On the stability of linear mappings in Banach spaces. Proc. Am. Math. Soc. 72, 297-300 (1978)

24. Tang, S, Zada, A, Faisal, S, El-Sheikh, MMA, Li, T: Stability of higher-order nonlinear impulsive differential equations. J. Nonlinear Sci. Appl. 9, 4713-4721 (2016)

25. Zada, A, Ali, W, Farina, S: Hyers-Ulam stability of nonlinear differential equations with fractional integrable impulses. Math. Methods Appl. Sci. (2017). doi:10.1002/mma.4405

26. Zada, A, Faisal, S, Li, Y: On the Hyers-Ulam stability of first-order impulsive delay differential equations. J. Funct. Spaces 2016, Article ID 8164978 (2016)

27. Zada, A, Faisal, S, Li, Y: Hyers-Ulam-Rassias stability of non-linear delay differential equations. J. Nonlinear Sci. Appl. 10 504-510 (2017)

28. Zada, A, Wang, P, Lassoued, D, Li, T: Connections between Hyers-Ulam stability and uniform exponential stability of 2-periodic linear nonautonomous systems. Adv. Differ. Equ. 2017, Article ID 192 (2017)

29. Zada, A, Shah, SO: Hyers-Ulam stability of first-order non-linear delay differential equations with fractional integrable impulses. Hacet. J. Math. Stat. (2017). doi:10.15672/HJMS.2017.496

30. Zada, A, Shah, SO, Shah, R: Hyers-Ulam stability of non-autonomous systems in terms of boundedness of Cauchy problem. Appl. Math. Comput. 271, 512-518 (2015)

31. Zada, A, Shah, R: A fixed point approach to the stability of a nonlinear Volterra integrodifferential equations with delay. Hacet. J. Math. Stat. (2017). doi:10.15672/HJMS.2017.467

32. Wang, J, Feckan, M, Zhou, Y: Ulam's type stability of impulsive ordinary differential equations. J. Math. Anal. Appl. 395 258-264 (2012)

33. Jung, SM: Hyers-Ulam-Rassias Stability of Functional Equations in Nonlinear Analysis. Springer, New York (2011)

34. Kilbas, AA, Srivastava, HM, Trujillo, JJ: Theory and Applications of Fractional Differential Equations. North-Holland Mathematics Studies, vol. 204. Elsevier, Amsterdam (2006)

35. Jung, CJ: On generalized complete metric spaces. Bull. Am. Math. Soc. 75(1), 113-116 (1969)

36. Diaz, JB, Margolis, B: A fixed point theorem of alternative, for contractions on a generalized complete metric space. Bull. Am. Math. Soc. 74, 305-309 (1968) 